\title{
Effective actions for dual massive (super) $p$-forms
}

\section{Sergei M. Kuzenko and Kai Turner}

Department of Physics M013, The University of Western Australia, 35 Stirling Highway, Perth W.A. 6009, Australia

E-mail: sergei.kuzenko@uwa.edu.au, 22226913@student.uwa.edu.au

ABSTRACT: In $d$ dimensions, the model for a massless $p$-form in curved space is known to be a reducible gauge theory for $p>1$, and therefore its covariant quantisation cannot be carried out using the standard Faddeev-Popov scheme. However, adding a mass term and also introducing a Stueckelberg reformulation of the resulting $p$-form model, one ends up with an irreducible gauge theory which can be quantised à la Faddeev and Popov. We derive a compact expression for the massive $p$-form effective action, $\Gamma_{p}^{(m)}$, in terms of the functional determinants of Hodge-de Rham operators. We then show that the effective actions $\Gamma_{p}^{(m)}$ and $\Gamma_{d-p-1}^{(m)}$ differ by a topological invariant. This is a generalisation of the known result in the massless case that the effective actions $\Gamma_{p}$ and $\Gamma_{d-p-2}$ coincide modulo a topological term. Finally, our analysis is extended to the case of massive super $p$-forms coupled to background $\mathcal{N}=1$ supergravity in four dimensions. Specifically, we study the quantum dynamics of the following massive super $p$-forms: (i) vector multiplet; (ii) tensor multiplet; and (iii) three-form multiplet. It is demonstrated that the effective actions of the massive vector and tensor multiplets coincide. The effective action of the massive threeform is shown to be a sum of those corresponding to two massive scalar multiplets, modulo a topological term.

KeYwords: Supergravity Models, Superspaces, Supersymmetric Effective Theories, Supersymmetry and Duality

ArXiv ePrint: 2009.08263

Dedicated to the memory of Professor Omar Foda 


\section{Contents}

1 Introduction 1

2 Massive $p$-forms in $d$ dimensions 5

2.1 Classical dynamics 5

2.2 Duality equivalence 6

2.3 Quantisation 7

2.4 Quantum equivalence 9

3 Massive $p$-forms in four dimensions $\quad 11$

3.1 Two-form field 11

3.2 Three-form field 12

4 Massive super $p$-forms in four dimensions $\quad 13$

$\begin{array}{lll}4.1 & \text { Setup } & 13\end{array}$

$\begin{array}{lll}4.2 & \text { Quantisation of the massive vector multiplet model } & 14\end{array}$

$\begin{array}{ll}4.3 & \text { Quantisation of the massive tensor multiplet model } \\ \end{array}$

4.4 Quantisation of the massive three-form multiplet model 16

$\begin{array}{ll}4.5 & \text { Analysis of the results } \\ \end{array}$

5 Discussion and generalisations $\quad 19$

A Hodge-de Rham operator 23

B Massless $p$-forms in $d$ dimensions $\quad 23$

C Massless three-form in four dimensions $\quad 24$

$\begin{array}{ll}\text { D Duality with topological mass term } & 25\end{array}$

\section{Introduction}

The model for a massless gauge two-form in four dimensions was introduced in the mid1960s by Ogievetsky and Polubarinov [1] who showed that it describes a spin-zero particle. Unfortunately, their work remained largely unknown for a decade. The same model was rediscovered, and generalised, twice in 1974 in the context of dual resonance models $[2,3]$. However, active studies of gauge $p$-forms in diverse dimensions began only in the late 1970s when it was recognised that such fields naturally occur in supergravity theories, see, e.g., [46] for early publications and [7-9] for reviews. Gauge $p$-forms are also of special interest in string theory where they appear in the low-energy effective actions see, e.g., [10-13] for reviews. 
There are two important themes in modern quantum field theory that originated by studying the quantum dynamics of massless gauge $p$-forms: (i) reducible gauge theories; and (ii) quantum equivalence of dual theories. It is appropriate here to briefly recall these developments.

For $p>1$, all massless $p$-form actions are examples of the so-called reducible gauge theories (following the terminology of the Batalin-Vilkovisky formalism [14]). In the framework of covariant Lagrangian quantisation, reducibility means that the generators of gauge transformations are linearly dependent. This fact has a number of non-trivial implications, which are: (i) gauge-fixing functions are constrained; (ii) ghosts for ghosts are required; and (iii) a naive application of the Faddeev-Popov quantisation scheme leads to incorrect results. Several consistent quantisation procedures have been developed to quantise reducible Abelian gauge theories such as gauge $p$-forms [15-19], including the formulations of $[17,19]$ which apply in the supersymmetric case. These quantisation schemes are much easier to deal with than the Batalin-Vilkovisky formalism [14]. ${ }^{1}$

In $d$ dimensions, two massless field theories describing a $p$-form and a $(d-p-2)$-form are known to be classically equivalent, see, e.g., [9, 23] for reviews. These theories are dual in the sense that the corresponding actions are related through the use of a firstorder (or parent) action, see e.g. [24]. The issue of quantum equivalence of such classically equivalent theories was raised, building on the results of [25], in 1980 by Duff and van Nieuwenhuizen [26, 27]. They showed, in particular, that (i) a massless two-form and a non-conformal scalar in four dimensions give rise to different trace anomalies; and (ii) the corresponding one-loop divergences differ by a topological term. These results were interpreted in [26] as a possible quantum non-equivalence of these dual field realisations. The issue was resolved in several publications $[19,24,28,29]$ in which it was shown that the effective actions of dual massless theories in four dimensions differ only by a topological invariant being independent of the spacetime metric. As a result, the dual theories are characterised by the same quantum energy-momentum tensor, $\left\langle T_{a b}\right\rangle$, which proves their quantum equivalence. ${ }^{2}$ Analogous results hold in higher dimensions [24, 30], as well as for dual supersymmetric field theories in four dimensions [19, 29] (see also [31] for a review). It is worth discussing the supersymmetric story in some more detail.

Several important massless $\mathcal{N}=1$ supermultiplets in four dimensions can be realised in terms of super $p$-forms [32] (see also [33]), with the cases $p=0,2$ and 3 corresponding to the chiral, tensor and three-form multiplets, respectively. The corresponding supersymmetric theories are related either by a duality transformation or by a superfield reparametrisation.

\footnotetext{
${ }^{1}$ One of the earliest applications of the Batalin-Vilkovisky formalism [14] was the Lagrangian quantisation [20, 21] of the Freedman-Townsend model [22]. Ref. [20] was accepted for publication in Sov. J. Nucl. Phys. in 1987. It was subsequently withdrawn shortly before publication, after the authors had been informed by a colleague that the same problem had already been solved elsewhere. Due to a limited access to the journals, at the time it was not possible to verify this information, which in fact turned out to be false.

${ }^{2}$ In the four-dimensional case, the dual two-form and zero-form theories are classically non-conformal. As emphasised in [29], the quantum operator $T^{a}{ }_{a}$ in such theories "contains the effects of both classical and quantum breaking and is not equal to the trace anomaly." In other words, there is no point to compare trace anomalies in classically non-conformal theories.
} 
The simplest model for the tensor multiplet [34] in a supergravity background is given by the action $^{3}$

$$
S_{\text {tensor }}[\Psi, \bar{\Psi}]=-\frac{1}{2} \int \mathrm{d}^{4} x \mathrm{~d}^{2} \theta \mathrm{d}^{2} \bar{\theta} E(G(\Psi))^{2}, \quad G(\Psi):=\frac{1}{2}\left(\mathcal{D}^{\alpha} \Psi_{\alpha}+\overline{\mathcal{D}}_{\dot{\alpha}} \bar{\Psi}^{\dot{\alpha}}\right),
$$

where $\Psi_{\alpha}$ is a covariantly chiral spinor, $\overline{\mathcal{D}}_{\dot{\beta}} \Psi_{\alpha}=0$. Its dual version [34]

$$
S_{\text {chiral }}[\Phi, \bar{\Phi}]=\frac{1}{2} \int \mathrm{d}^{4} x \mathrm{~d}^{2} \theta \mathrm{d}^{2} \bar{\theta} E(\Phi+\bar{\Phi})^{2}, \quad \overline{\mathcal{D}}_{\dot{\beta}} \Phi=0,
$$

describes the non-conformal scalar multiplet.

Let us represent the dynamical variables in (1.2) as $\Phi=\mathcal{P}_{+} V$ and $\bar{\Phi}=\mathcal{P}_{-} V$, where $V$ is a real scalar and the operators $\mathcal{P}_{+}$and $\mathcal{P}_{-}$have the form ${ }^{4}$

$$
\mathcal{P}_{+}=-\frac{1}{4}\left(\overline{\mathcal{D}}^{2}-4 R\right), \quad \mathcal{P}_{-}=-\frac{1}{4}\left(\mathcal{D}^{2}-4 \bar{R}\right) .
$$

Then we end up with the three-form multiplet realisation [32] of the non-conformal scalar multiplet. The corresponding action is

$$
S_{3 \text {-form }}[V]=\frac{1}{2} \int \mathrm{d}^{4} x \mathrm{~d}^{2} \theta \mathrm{d}^{2} \bar{\theta} E V\left(\mathcal{P}_{+}+\mathcal{P}_{-}\right)^{2} V, \quad \bar{V}=V .
$$

This theory was studied in [19], see also [31] for a review.

The models (1.1) and (1.2) are dually equivalent [34]. Their quantum equivalence was established in [29] in the case of an on-shell supergravity background, and in [19] for an arbitrary supergravity background.

Since the three-form multiplet action (1.4) is obtained from (1.2) by setting $\Phi=\mathcal{P}_{+} V$, the physical fields can be chosen to coincide in both models. The main difference between the models (1.2) and (1.4) at the component level is that one of the two real auxiliary scalars in (1.2) is replaced by (the Hodge dual of) the field strength of a three-form in the case of (1.4). Being non-dynamical, the three-form is known to generate a positive contribution to the cosmological constant [26, 37-41]. In order to achieve a better understanding of the three-form multiplet model (1.4), we describe its dual version. It is obtained by starting with the first-order action [19]

$$
S[V, L]=-\int \mathrm{d}^{4} x \mathrm{~d}^{2} \theta \mathrm{d}^{2} \bar{\theta} E\left\{\frac{1}{2} L^{2}-L\left(\mathcal{P}_{+}+\mathcal{P}_{-}\right) V\right\},
$$

where $V$ and $L$ are unconstrained real scalars. Varying $S[V, L]$ with respect to $L$ leads to the three-form multiplet action (1.4). On the other hand, varying $V$ gives

$$
\left(\mathcal{P}_{+}+\mathcal{P}_{-}\right) L=0 \quad \Longrightarrow \quad \mathcal{P}_{+} L=\mathrm{i} \mu, \quad \mu=\bar{\mu}=\text { const } .
$$

\footnotetext{
${ }^{3}$ Our consideration below can readily be extended to the nonlinear theories which were introduced in [34] and are obtained by replacement $G^{2} \rightarrow f(G)$. However, such theories are non-renormalisable in general and will not be studied in what follows. It should be pointed out that the duality transformations for the nonlinear $f(G)$ models were described in [35]. The special choice of $f(G) \propto G \ln G$ corresponds to the so-called improved (superconformal) tensor multiplet [36].

${ }^{4}$ For any scalar superfield $U, \mathcal{P}_{+} U$ is covariantly chiral, and $\mathcal{P}_{-} U$ antichiral.
} 
This constraint defines a deformed tensor multiplet, in accordance with the terminology of [42]. The dynamics of this multiplet is described by the action

$$
S[\mathfrak{L}]=-\frac{1}{2} \int \mathrm{d}^{4} x \mathrm{~d}^{2} \theta \mathrm{d}^{2} \bar{\theta} E \mathfrak{L}^{2}, \quad \mathcal{P}_{+} \mathfrak{L}=\mathrm{i} \mu, \quad \mu=\bar{\mu}=\text { const } .
$$

At the component level, the main manifestation of the deformation parameter $\mu$ in (1.7) is the emergence of a positive cosmological constant. Unlike (1.7), no parameter $\mu$ is present in the action (1.4). However, $\mu$ gets generated dynamically, since the general solution of the equation of motion for (1.4) contains such a parameter,

$$
\left(\mathcal{P}_{+}+\mathcal{P}_{-}\right)^{2} V=0 \quad \Longrightarrow \quad \mathcal{P}_{+}\left(\mathcal{P}_{+}+\mathcal{P}_{-}\right) V=\mathrm{i} \mu,
$$

with $\mu$ a real parameter. On the mass shell, we can identify $\left(\mathcal{P}_{+}+\mathcal{P}_{-}\right) V=\mathfrak{L}$. The effective actions corresponding to different values of $\mu$ differ by a cosmological term. The authors of [19] made use of the choice $\mu=0$ and demonstrated that the effective actions $\Gamma_{\text {chiral }}$ and $\Gamma_{3 \text {-form, }}$ which correspond to the locally supersymmetric models (1.2) and (1.4), differ by a topological invariant.

It should be pointed out that general duality transformations with three-form multiplets and their applications were studied in [43-45].

So far we have discussed the models for massless $p$-forms and their supersymmetric extensions. Massive antisymmetric tensor fields were discussed in the physics literature even earlier than the massless ones. Kemmer in 1960 [46], and independently Takahashi and Palmer in 1970 [47], showed that the massive spin-1 particle can be described using a 2-form field. Further publications on massive antisymmetric fields [3, 48-53] revealed, in particular, that a massive $p$-form in $d$ dimensions is dual to a massive $(d-p-1)$-form. ${ }^{5}$ This raised the issue of quantum equivalence of dual models. Some quantum aspects of massive $p$-forms were studied using the worldline approach in $[56,57]$. In the important work by Buchbinder, Kirillova and Pletnev [58], the quantum equivalence of classically equivalent massive $p$-forms in four dimensions was established. In the present work we extend the results of [58] to $d$ dimensions. Our proof of the quantum equivalence of dual theories in $d=4$ differs from the one given in [58]. Our approach is also extended to the case of massive super $p$-forms coupled to background $\mathcal{N}=1$ supergravity in four dimensions. Specifically, we study the quantum dynamics of the following massive super $p$-forms: (i) vector multiplet; (ii) tensor multiplet; and (iii) three-form multiplet. In particular, we demonstrate that the effective actions of the massive vector and tensor multiplets coincide.

Massive super $p$-forms have recently found numerous applications, including the effective description of gaugino condensation [59-62], inflationary cosmology [63], and effective field theories from string flux compactifications [64] (see also [65] for a review). Here we do not attempt to give a complete list of works on massive super $p$-forms and their applications. However it is worth mentioning those publications in which such supermultiplets were introduced in the case of four dimensional $\mathcal{N}=1$ supersymmetry. Massive tensor and vector multiplets coupled to supergravity were studied in $[34,53,66]$. Tensor multiplets

\footnotetext{
${ }^{5}$ Massive $p$-forms naturally occur in the framework of string compactifications with non-trivial background fluxes [54, 55].
} 
with complex masses were studied in [69-71]. To the best of our knowledge, a massive three-form multiplet was first discussed in [31], although a massive three-form is contained at the component level in one of the models introduced by Gates and Siegel [72].

This paper is organised as follows. In section 2 we derive effective actions $\Gamma_{p}^{(m)}$ for massive $p$-form models in $d$-dimensional curved spacetime. We then demonstrate that, for $0 \leq p \leq d-1$, the effective actions $\Gamma_{p}^{(m)}$ and $\Gamma_{d-p-1}^{(m)}$ differ by a topological invariant. Section 3 is devoted to alternative proofs of some of the results of section 2 specifically for the $d=4$ case. Effective actions for massive super $p$-forms in four dimensions are studied in section 4 . In section 5 we discuss the obtained results and sketch several generalisations. Four technical appendices are included. Appendix A collects the properties of the Hodge-de Rham operator. Appendix B gives a summary of the results concerning massless $p$-forms in $d$ dimensions. The effective action of a massless three-form in $d=4$ is discussed in appendix C. Finally, appendix D describes dual formulations in the presence of a topological mass term. We make use of the Grimm-Wess-Zumino geometry [73] which underlies the WessZumino formulation [74] for old minimal supergravity (see [75] for a review) discovered independently in [76-81]. Our two-component spinor notation and conventions follow [31]. The algebra of the supergravity covariant derivatives, which we use, is given in section 5.5.3 of [31].

In order to have a uniform notation for non-supersymmetric and supersymmetric theories, in this paper we make use of the vielbein formulation for gravity. The background gravitational field is described by a vielbein $e^{a}=\mathrm{d} x^{m} e_{m}{ }^{a}(x)$, such that $e=\operatorname{det}\left(e_{m}{ }^{a}\right) \neq 0$, and the metric is a composite field defined by $g_{m n}=e_{m}{ }^{a} e_{n}{ }^{b} \eta_{a b}$, with $\eta_{a b}$ the Minkowski metric. All $p$-form fields in $d$ dimensions carry Lorentz indices. We make use of the torsion-free covariant derivatives

$$
\nabla_{a}=e_{a}+\omega_{a}=e_{a}^{m} \partial_{m}+\frac{1}{2} \omega_{a}^{b c} M_{b c}, \quad\left[\nabla_{a}, \nabla_{b}\right]=\frac{1}{2} R_{a b}^{c d} M_{c d} .
$$

Here $M_{b c}=-M_{c b}$ denotes the Lorentz generators, $e_{a}{ }^{m}(x)$ the inverse vielbein, $e_{a}{ }^{m} e_{m}{ }^{b}=$ $\delta_{a}{ }^{b}$. The Lorentz generators act on a $d$-vector $v^{a}$ as $M_{b c} v^{a}=\delta^{a}{ }_{b} v_{c}-\delta^{a}{ }_{c} v_{b}=2 \delta^{a}{ }_{[b} b_{c]}$.

\section{Massive $p$-forms in $d$ dimensions}

In this section we derive effective actions $\Gamma_{p}^{(m)}$ for massive $p$-form models in curved space and demonstrate that $\Gamma_{p}^{(m)}$ and $\Gamma_{d-p-1}^{(m)}$ differ by a topological invariant.

\subsection{Classical dynamics}

Let $B_{a_{1} \ldots a_{p}}(x)=B_{\left[a_{1} \ldots a_{p}\right]}(x) \equiv B_{a(p)}(x)$ be a differential $p$-form in curved space $\mathcal{M}^{d}$. The dynamics of a massive $p$-form is described by the action

$$
\begin{aligned}
S_{p}^{(m)}[B]= & -\frac{1}{2(p+1) !} \int \mathrm{d}^{d} x \text { e } F^{a_{1} \ldots a_{p+1}}(B) F_{a_{1} \ldots a_{p+1}}(B) \\
& -\frac{m^{2}}{2 p !} \int \mathrm{d}^{d} x \text { e } B^{a_{1} \ldots a_{p}} B_{a_{1} \ldots a_{p}},
\end{aligned}
$$


where $F_{a_{1} \ldots a_{p+1}}(B):=(p+1) \nabla_{\left[a_{1}\right.} B_{\left.a_{2} \ldots a_{p+1}\right]}$ is the field strength, and $m$ the mass. It is assumed in this section that $m \neq 0$. The Euler-Lagrange equation corresponding to (2.1) is

$$
\nabla^{b} F_{b a_{1} \ldots a_{p}}(B)-m^{2} B_{a_{1} \ldots a_{p}}=0 \text {. }
$$

It implies that

$$
\nabla^{c} B_{c a_{1} \ldots a_{p-1}}=0
$$

and therefore the equation of motion turns into

$$
\left(\square_{p}-m^{2}\right) B_{a_{1} \ldots a_{p}}=0,
$$

where $\square_{p}$ is the covariant d'Alembertian (A.5).

The symmetric energy-momentum tensor corresponding to the model (2.1) is

$$
\begin{aligned}
T_{[p, m]}^{a b}(B)= & \frac{1}{p !}\left\{F^{a c_{1} \ldots c_{p}}(B) F_{c_{1} \ldots c_{p}}^{b}(B)-\frac{1}{2(p+1)} \eta^{a b} F^{c_{1} \ldots c_{p+1}}(B) F_{c_{1} \ldots c_{p+1}}(B)\right\} \\
& +\frac{m^{2}}{(p-1) !}\left\{B^{a c_{1} \ldots c_{p-1}} B^{b}{ }_{c_{1} \ldots c_{p-1}}-\frac{1}{2 p} \eta^{a b} B^{c_{1} \ldots c_{p}} B_{c_{1} \ldots c_{p}}\right\}
\end{aligned}
$$

with $\eta_{a b}$ the Minkowski metric. It is conserved,

$$
\nabla_{b} T_{[p, m]}^{a b}=0
$$

on the mass shell.

\subsection{Duality equivalence}

It is known that the massless models for a $p$-form and $(d-p-2)$-form are classically equivalent, see appendix B. In the massive case, however, a $p$-form is dual to a $(d-p-1)$ form, see, e.g., [51, 53]. Here we recall the proof of this result. To demonstrate that the massive theories with actions $S_{p}^{(m)}[B]$ and $S_{d-p-1}^{(m)}[A]$ are equivalent, we first rewrite (2.1) in the form

$$
\begin{aligned}
S_{p}^{(m)}[B]= & \frac{1}{2(d-p-1) !} \int \mathrm{d}^{d} x e L^{a(d-p-1)}(B) L_{a(d-p-1)}(B) \\
& -\frac{m^{2}}{2 p !} \int \mathrm{d}^{d} x \text { e } B^{a(p)} B_{a(p)},
\end{aligned}
$$

where

$$
L^{a_{1} \ldots a_{d-p-1}}(B):=\frac{1}{(p+1) !} \varepsilon^{a_{1} \ldots a_{d-p-1} b_{1} \ldots b_{p+1}} F_{b_{1} \ldots b_{p+1}}(B) \equiv * F^{a_{1} \ldots a_{d-p-1}}(B),
$$

and introduce the first-order action

$$
\begin{aligned}
S\left[B_{p}, L_{q}, A_{q}\right]=\int \mathrm{d}^{d} x e\left\{\frac{1}{2 q !} L^{a(q)} L_{a(q)}-\frac{m^{2}}{2 p !} B^{a(p)} B_{a(p)}\right. \\
\left.+\frac{m}{q !} A_{a(q)}\left(L^{a(q)}-* F^{a(q)}(B)\right)\right\}, \quad q=d-p-1 .
\end{aligned}
$$


Here the variables $L_{q}$ and $A_{q}$ are unconstrained $(d-p-1)$-forms. Varying with respect to $A_{q}$ returns the original action, eq. (2.1). On the other hand, varying with respect to $L_{q}$ and $B_{p}$ leads to the dual action $S_{d-p-1}^{(m)}[A]$.

The equations of motion corresponding to (2.8) are

$$
\begin{aligned}
m B_{a_{1} \ldots a_{p}} & =-(-1)^{d(p+1)} * F_{a_{1} \ldots a_{p}}(A), \\
m A_{a_{1} \ldots a_{q}} & :=-L_{a_{1} \ldots a_{q}}, \\
L_{a_{1} \ldots a_{q}} & =* F_{a_{1} \ldots a_{q}}(B) .
\end{aligned}
$$

Making use of these equations, one may show that the energy-momentum tensors in the theories $S_{p}^{(m)}[B]$ and $S_{d-p-1}^{(m)}[A]$ coincide,

$$
T_{[p, m]}^{a b}(B)=T_{[d-p-1, m]}^{a b}(A) .
$$

\subsection{Quantisation}

Associated with the massive $p$-form model (2.1) is the effective action $\Gamma_{p}^{(m)}$ defined by

$$
\mathrm{e}^{\mathrm{i} \Gamma_{p}^{(m)}}=\int\left[\mathcal{D} B_{p}\right] \mathrm{e}^{\mathrm{i} S_{p}^{(m)}[B]} .
$$

To obtain a useful expression for $\Gamma_{p}^{(m)}$, we introduce a Stueckelberg reformulation of the theory. It is obtained from (2.1) by replacing

$$
B_{a_{1} \ldots a_{p}} \rightarrow B_{a_{1} \ldots a_{p}}+\frac{1}{m} F_{a_{1} \ldots a_{p}}(V)
$$

for some $(p-1)$-form $V_{a_{1} \ldots a_{p-1}}(x)$. The resulting action

$$
\begin{aligned}
S_{p}^{(m)}[B, V]= & -\frac{1}{2(p+1) !} \int \mathrm{d}^{d} x \text { e } F^{a(p+1)}(B) F_{a(p+1)}(B)-\frac{m^{2}}{2 p !} \int \mathrm{d}^{d} x \text { e } B^{a(p)} B_{a(p)} \\
& -\frac{1}{2 p !} \int \mathrm{d}^{d} x \text { e } F^{a(p)}(V) F_{a(p)}(V)-\frac{m}{p !} \int \mathrm{d}^{d} x \text { e } B^{a(p)} F_{a(p)}(V)
\end{aligned}
$$

is invariant under gauge transformations

$$
\delta_{\zeta} B_{a(p)}=p \nabla_{\left[a_{1}\right.} \zeta_{\left.a_{2} \ldots a_{p}\right]} \equiv F_{a(p)}(\zeta), \quad \delta_{\zeta} V_{a(p-1)}=-m \zeta_{a(p-1)} .
$$

The gauge freedom allows us to choose the gauge condition $V_{a(p-1)}=0$ and then we are back to the original model.

The compensating field $V_{a(p-1)}$ appears in the action (2.13) only via the field strength $F_{a(p)}(V)$ which is invariant under gauge transformations

$$
\delta_{\lambda} V_{a(p-1)}=(p-1) \nabla_{\left[a_{1}\right.} \lambda_{\left.a_{2} \ldots a_{p-1}\right]} \equiv F_{a(p-1)}(\lambda) .
$$

This gauge freedom is characterised by linearly dependent generators, which makes it tempting to conclude that the gauge theory under consideration is reducible. Nevertheless, (2.13) is an irreducible gauge theory and can be quantised à la Faddeev and Popov. 
The point is that (2.15) is a special case of the transformation (2.14) with $\zeta_{a(p-1)}=$ $-m^{-1} F_{a(p-1)}(\lambda)$.

To quantise the gauge theory with action (2.13), we choose the gauge fixing

$$
\chi_{a(p-1)}=\nabla^{b} B_{b a(p-1)}+m V_{a(p-1)}-\rho_{a(p-1)},
$$

with $\rho_{a(p-1)}$ an external field. The gauge variation of $\chi_{a(p-1)}$ is

$$
\delta_{\zeta} \chi_{a(p-1)}=\nabla^{b} F_{b a(p-1)}(\zeta)-m^{2} \zeta_{a(p-1)} \equiv(\mathfrak{O} \zeta)_{a(p-1)} .
$$

Here $\mathfrak{O}$ is the kinetic operator in the massive $p$-form model (2.1). Making use of (2.11), we conclude that the Faddeev-Popov determinant $\Delta_{\mathrm{FP}}$ is

$$
\Delta_{\mathrm{FP}}=\operatorname{det} \mathfrak{O}=\exp \left(-2 \mathrm{i} \Gamma_{p-1}^{(m)}\right) .
$$

Now, in accordance with the Faddeev-Popov procedure, the effective action is

$$
\mathrm{e}^{\mathrm{i} \Gamma_{p}^{(m)}}=\int\left[\mathcal{D} B_{p}\right]\left[\mathcal{D} V_{p-1}\right] \operatorname{det} \mathfrak{O} \delta\left[\nabla^{b} B_{b a(p-1)}+m V_{a(p-1)}-\rho_{a(p-1)}\right] \mathrm{e}^{\mathrm{i} S_{p}^{(m)}[B, V]} .
$$

Averaging the right-hand side over $\rho_{a(p-1)}$ with weight

$$
\exp \left\{-\frac{\mathrm{i}}{2(p-1) !} \int \mathrm{d}^{d} x e \rho^{a(p-1)} \rho_{a(p-1)}\right\}
$$

leads to

$$
\begin{aligned}
\mathrm{e}^{\mathrm{i} \Gamma_{p}^{(m)}} & =\int\left[\mathcal{D} B_{p}\right]\left[\mathcal{D} V_{p-1}\right] \operatorname{det} \mathfrak{O} \mathrm{e}^{\mathrm{i} S_{\text {quant }}[B, V]}, \\
S_{\text {quant }}[B, V] & =\frac{1}{2 p !} \int \mathrm{d}^{d} x e B^{a(p)}\left(\square_{p}-m^{2}\right) B_{a(p)}+S_{p-1}^{(m)}[V] .
\end{aligned}
$$

As a result, for the effective action we obtain

$$
\exp \left\{\mathrm{i} \Gamma_{p}^{(m)}\right\}=\exp \left\{-\mathrm{i} \Gamma_{p-1}^{(m)}\right\}\left[\operatorname{det}\left(\square_{p}-m^{2}\right)\right]^{-\frac{1}{2}} .
$$

This is a recurrence relation. It leads to a simple expression for the effective action

$$
\Gamma_{p}^{(m)}=\frac{\mathrm{i}}{2} \sum_{k=0}^{p}(-1)^{k} \ln \operatorname{det}\left(\square_{p-k}-m^{2}\right)=\frac{\mathrm{i}}{2}(-1)^{p} \sum_{k=0}^{p}(-1)^{k} \ln \operatorname{det}\left(\square_{k}-m^{2}\right) .
$$

In the $d=4$ case, this result agrees with [58].

The representation (2.23) is formal since each term on the right-hand side contains UV divergences. This issue is addressed by introducing a regularisation for the effective

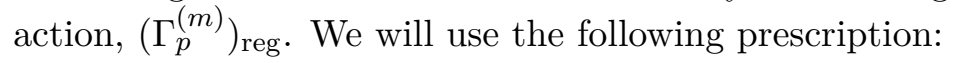

$$
\left(\Gamma_{p}^{(m)}\right)_{\mathrm{reg}}=-\frac{\mathrm{i}}{2}(-1)^{p} \int_{0}^{\infty} \frac{\mathrm{d} s}{s^{1-\omega}} \mathrm{e}^{-\mathrm{i}\left(m^{2}-\mathrm{i} \varepsilon\right) s} \sum_{k=0}^{p}(-1)^{k} \int \mathrm{d}^{d} x e \operatorname{tr} \mathfrak{U}_{k}(x, x \mid s),
$$

with $\omega, \varepsilon \rightarrow+0$. Here the right-hand side involves the (heat) kernel of the evolution operator $\mathfrak{U}_{k}(s)=\exp \left(\mathrm{i} s \square_{k}\right)$ acting on the space of $k$-forms. The kernel of $\mathfrak{U}_{k}(s)$ is defined by

$$
\mathfrak{U}_{a(k)}{ }^{a^{\prime}(k)}\left(x, x^{\prime} \mid s\right)=\mathrm{e}^{\mathrm{i} s \square_{k}} \delta_{a(k)} a^{\prime}(k)\left(x, x^{\prime}\right),
$$


where the delta-function is

$$
\delta_{a(k)}{ }^{a^{\prime}(k)}\left(x, x^{\prime}\right)=k ! \delta_{\left[a_{1}\right.}{ }^{a_{1}^{\prime}} \ldots \delta_{\left.a_{k}\right]}{ }^{a_{k}^{\prime}} e^{-1} \delta^{d}\left(x-x^{\prime}\right)=k ! \delta_{a_{1}}{ }^{\left[a_{1}^{\prime}\right.} \ldots \delta_{a_{k}}{ }^{\left.a_{k}^{\prime}\right]} e^{-1} \delta^{d}\left(x-x^{\prime}\right),
$$

such that

$$
\frac{1}{k !} \int \mathrm{d}^{d} x^{\prime} e\left(x^{\prime}\right) \delta_{a(k)}{ }^{a^{\prime}(k)}\left(x, x^{\prime}\right) \omega_{a^{\prime}(k)}\left(x^{\prime}\right)=\omega_{a(k)}(x),
$$

for any $k$-form $\omega$. In accordance with the definition of the delta-function, the trace over Lorentz indices in (2.24) is

$$
\operatorname{tr} \mathfrak{U}_{k}(x, x \mid s)=\frac{1}{k !} \mathfrak{U}_{a(k)}^{a(k)}(x, x \mid s) .
$$

\subsection{Quantum equivalence}

In $d$ dimensions, the model for a massive $p$-form is classically equivalent to that for a massive $(d-p-1)$-form. Let us analyse whether this equivalence extends to the quantum theory. Our analysis will be based on the fact that the spaces of $p$-forms and $(d-p)$-forms are isomorphic, and the corresponding Hodge d'Alembertians are related to each other as follows

$$
*\left(\square_{p} \omega\right)=\square_{d-p}(* \omega),
$$

where $\omega$ is an arbitrary $p$-form.

Making use of the relations (2.23) and (2.29), one may show that

$$
\Gamma_{p}^{(m)}-\Gamma_{d-p-1}^{(m)}=(-1)^{p} \mathfrak{X}^{(m)}, \quad \mathfrak{X}^{(m)}:=\frac{\mathrm{i}}{2} \sum_{k=0}^{d}(-1)^{k} \ln \operatorname{det}\left(\square_{k}-m^{2}\right) .
$$

There are two distinct cases. If the dimension of space-time is odd, $d=2 n+1$, the functional $\mathfrak{X}^{(m)}$ can be seen to vanish identically,

$$
d=2 n+1 \quad \Longrightarrow \quad \mathfrak{X}^{(m)}=0 .
$$

In the even-dimensional case, $d=2 n, \mathfrak{X}^{(m)}$ can be rewritten in the form:

$$
\mathfrak{X}^{(m)}=\mathrm{i} \sum_{k=0}^{d / 2-1}(-1)^{k} \ln \operatorname{det}\left(\square_{k}-m^{2}\right)+\frac{\mathrm{i}}{2}(-1)^{d / 2} \ln \operatorname{det}\left(\square_{d / 2}-m^{2}\right) .
$$

This functional is no longer identically zero. However, it turns out to be a topological invariant in the sense that

$$
\frac{\delta}{\delta e_{m}^{a}(x)} \mathfrak{X}^{(m)}=0
$$

In order to prove $(2.32)$, let us consider the regularised version of $\mathfrak{X}^{(m)}$

$$
\left(\mathfrak{X}^{(m)}\right)_{\text {reg }}=\frac{1}{2} \int_{0}^{\infty} \frac{\mathrm{d} s}{s^{1-\omega}} \mathrm{e}^{-\mathrm{i}\left(m^{2}-\mathrm{i} \varepsilon\right) s} \Upsilon(s), \quad \omega, \varepsilon \rightarrow+0,
$$


where we have introduced the functional

$$
\Upsilon(s)=-\mathrm{i} \sum_{k=0}^{d}(-1)^{k} \int \mathrm{d}^{d} x \text { etr } \mathfrak{U}_{k}(x, x \mid s) .
$$

Giving the gravitational field a small disturbance, the functional $\Upsilon(s)$ varies as

$$
\delta \Upsilon(s)=-\left.s \sum_{k=0}^{d}(-1)^{k} \int \mathrm{d}^{d} x e \operatorname{tr}\left\{\left(\mathrm{d} \delta \mathrm{d}^{\dagger}+\delta \mathrm{d}^{\dagger} \mathrm{d}\right) \mathfrak{U}_{k}\left(x, x^{\prime} \mid s\right)\right\}\right|_{x=x^{\prime}}
$$

This variation may be rearranged by making use of the Ward identities

$$
\begin{aligned}
(k+1) & \nabla_{\left[a_{1}\right.} \mathfrak{U}_{\left.a_{2} \ldots a_{k+1}\right]}{ }^{a^{\prime}(k)}\left(x, x^{\prime} \mid s\right)=-\nabla_{b^{\prime}} \mathfrak{U}_{a(k+1)}{ }^{b^{\prime} a^{\prime}(k)}\left(x, x^{\prime} \mid s\right), \\
-\nabla^{b} \mathfrak{U}_{b a_{1} \ldots a_{k-1}}{ }^{a^{\prime}(k)}\left(x, x^{\prime} \mid s\right) & =k \nabla^{\left[a_{1}^{\prime} \mathfrak{U}_{a(k-1)} a_{2}^{\prime} \ldots a_{k}^{\prime}\right]}\left(x, x^{\prime} \mid s\right),
\end{aligned}
$$

in conjunction with the relations

$$
\begin{array}{r}
\int \mathrm{d}^{d} x e\left\{\nabla^{b} \nabla_{[b} \mathfrak{U}_{a(k)]}{ }^{a^{\prime}(k)}\left(x, x^{\prime} \mid s\right)+\nabla^{\left[a_{k+1}^{\prime}\right.} \nabla_{\left[a_{k+1}\right.} \mathfrak{U}_{a(k)]}{ }^{\left.a^{\prime}(k)\right]}\left(x, x^{\prime} \mid s\right)\right\} \|=0, \\
\int \mathrm{d}^{d} x e\left\{k \nabla_{\left[a_{1}\right.} \nabla^{b} \mathfrak{U}_{\left.|b| a_{2} \ldots a_{k-1}\right]}{ }^{\prime}(k)\left(x, x^{\prime} \mid s\right)+\nabla_{b^{\prime}} \nabla^{b} \mathfrak{U}_{b a(k-1)}{ }^{b^{\prime} a^{\prime}(k-1)}\left(x, x^{\prime} \mid s\right)\right\} \|=0,
\end{array}
$$

where the double vertical bar means setting $x=x^{\prime}$ and $a=a^{\prime}$. Then one obtains

$$
\delta \Upsilon(s)=0
$$

which is equivalent to $(2.32)$.

Similar arguments may be used to show that $\Upsilon(s)$ is actually $s$-independent,

$$
\frac{\mathrm{d}}{\mathrm{d} s} \Upsilon(s)=-\left.\sum_{k=0}^{d}(-1)^{k} \int \mathrm{d}^{d} x e \operatorname{tr}\left\{\left(\mathrm{dd}^{\dagger}+\mathrm{d}^{\dagger} \mathrm{d}\right) \mathfrak{U}_{k}\left(x, x^{\prime} \mid s\right)\right\}\right|_{x=x^{\prime}}=0
$$

For small values of $s$, it is well known that the diagonal heat kernel has the asymptotic expansion

$$
\operatorname{tr} \mathfrak{U}_{k}(x, x \mid s)=\frac{\mathrm{i}}{(4 \pi \mathrm{i} s)^{d / 2}} \sum_{n=0}^{\infty}(\mathrm{i} s)^{n} \operatorname{tr} \mathfrak{a}_{k}^{(n)}(x, x),
$$

with $a_{n}(x, x)$ the Seeley-DeWitt coefficients. As a result, the topological invariant $(2.33 \mathrm{~b})$ takes the form

$$
\Upsilon=\frac{1}{(4 \pi)^{d / 2}} \sum_{k=0}^{d}(-1)^{k} \int \mathrm{d}^{d} x e \operatorname{tr} \mathfrak{a}_{k}^{(d / 2)}(x, x),
$$

which is the heat kernel expression for the Euler characteristic, see, e.g., [83].

The above analysis is a variant of the famous heat kernel proofs of the Chern-GaussBonnet theorem, see [83] for a review. 


\section{Massive $p$-forms in four dimensions}

In this section we will present alternative proofs of some results from the previous section in the $d=4$ case. The topological mismatch $\mathfrak{X}^{(m)}$ in $(2.30)$ will be ignored.

\section{$3.1 \quad$ Two-form field}

The model for a massive two-form in curved space is described by the action

$$
S_{2}^{(m)}[B]=\frac{1}{2} \int \mathrm{d}^{4} x e\left\{L^{a}(B) L_{a}(B)-\frac{m^{2}}{2} B^{a b} B_{a b}\right\},
$$

where we have denoted

$$
L^{a}(B)=\frac{1}{2} \varepsilon^{a b c d} \nabla_{b} B_{c d}=\frac{1}{6} \varepsilon^{a b c d} F_{b c d}(B) .
$$

This theory is classically equivalent to the model with action $S_{1}^{(m)}[V]$, which describes the massive vector field in curved space.

We are going to show that

$$
\exp \left(\mathrm{i} \Gamma_{2}^{(m)}\right)=\exp \left(\mathrm{i} \Gamma_{1}^{(m)}\right) .
$$

For this we consider the following change of variables ${ }^{6}$

$$
\begin{aligned}
B_{a b} & =2 \nabla_{[a} V_{b]}+\varepsilon_{a b c d} \nabla^{c} K^{d}, \\
\Phi & =\nabla_{a} K^{a}, \quad \Psi=\nabla_{a} V^{a} .
\end{aligned}
$$

Its Jacobian proves to be

$$
J(B, \Phi, \Psi \mid V, K)=\operatorname{det} \square_{1} .
$$

We perform the change of variables (3.4) in the action

$$
S[B, \Phi, \Psi]=S_{2}^{(m)}[B]-\frac{1}{2} \int \mathrm{d}^{4} x e \Phi\left(\square_{0}-m^{2}\right) \Phi-\frac{m^{2}}{2} \int \mathrm{d}^{4} x e \Psi^{2} .
$$

Then $S[B, \Phi, \Psi]$ turns into

$$
\frac{m^{2}}{2} \int \mathrm{d}^{4} x e V^{a} \square_{1} V_{a}+\frac{1}{2} \int \mathrm{d}^{4} x e K^{a}\left(\square_{1}-m^{2}\right) \square_{1} K_{a} .
$$

Making use of (3.5) leads to

$$
\begin{aligned}
\exp \left(\mathrm{i} \Gamma_{2}^{(m)}\right)\left[\operatorname{det}\left(\square_{0}-m^{2}\right)\right]^{-1 / 2} & =\int\left[\mathcal{D} B_{2}\right][\mathcal{D} \Phi][\mathcal{D} \Psi] \exp (\mathrm{i} S[B, \Phi, \Psi]) \\
& =\left[\operatorname{det}\left(\square_{1}-m^{2}\right)\right]^{-1 / 2}
\end{aligned}
$$

which is equivalent to (3.3).

\footnotetext{
${ }^{6}$ Given an arbitrary $p$-form $\omega_{p}$ on a compact Riemannian manifold $(M, g)$, the Hodge decomposition theorem states that $\omega_{p}=\mathrm{d} \varphi_{p-1}+\mathrm{d}^{\dagger} \Psi_{p+1}+h_{p}$, where $h_{p}$ is harmonic, $\square_{p} h_{p}=0$. It is assumed in (3.4a) that $\square_{p}$ has no normalised zero modes.
} 


\subsection{Three-form field}

The model for a massive three-form in curved space is described by the action

$$
S_{3}^{(m)}[V]=\frac{1}{2} \int \mathrm{d}^{4} x e\left\{\left(\nabla_{a} V^{a}\right)^{2}+m^{2} V^{a} V_{a}\right\} .
$$

In terms of the field strength $H=\nabla_{a} V^{a}$, the equation of motion is

$$
\nabla_{a} H-m^{2} V_{a}=0 \quad \Longrightarrow \quad\left(\square_{0}-m^{2}\right) H=0 .
$$

This shows that the three-form model (3.9) is equivalent to the massive scalar model

$$
S_{0}^{(m)}[\varphi]=-\frac{1}{2} \int \mathrm{d}^{4} x e\left\{\nabla^{a} \varphi \nabla_{a} \varphi+m^{2} \varphi^{2}\right\} .
$$

Classical equivalence of the theories (3.9) and (3.11) is established by considering a firstorder model with Lagrangian

$$
\mathcal{L}=\rho \nabla_{a} V^{a}-\frac{1}{2} \rho^{2}+\frac{1}{2} m^{2} V^{a} V_{a} .
$$

The effective action for the massive three-form model is

$$
\exp \left(\mathrm{i} \Gamma_{3}^{(m)}\right)=\int[\mathcal{D} V] \mathrm{e}^{\mathrm{i} S_{3}^{(m)}[V]} .
$$

We are going to show that

$$
\exp \left(\mathrm{i} \Gamma_{3}^{(m)}\right)=\exp \left(\mathrm{i} \Gamma_{0}^{(m)}\right) .
$$

For this we consider the following change of variables [19]

$$
\begin{aligned}
& V_{a}=\nabla_{a} \Phi+\frac{1}{2} \varepsilon_{a b c d} \nabla^{b} B^{c d} \equiv \nabla_{a} \Phi+L_{a}(B), \\
& K_{a}=\nabla_{a} \Psi+\nabla^{b} B_{a b} \equiv \nabla_{a} \Psi+G_{a}(B) .
\end{aligned}
$$

The corresponding Jacobian is

$$
J(V, K \mid B, \Phi, \Psi)=\operatorname{det} \square_{0}\left(\operatorname{det} \square_{2}\right)^{\frac{1}{2}},
$$

see [19] for the derivation. We perform the above change of variables in the path integral

$$
\exp \left(2 \mathrm{i} \Gamma_{3}^{(m)}\right)=\int[\mathcal{D} V][\mathcal{D} K] \operatorname{expi}\left(S_{3}^{(m)}[V]-S_{3}^{(m)}[K]\right)
$$

For the action $S_{3}^{(m)}[V]-S_{3}^{(m)}[K]$ we obtain

$$
\begin{aligned}
S_{3}^{(m)}[V]-S_{3}^{(m)}[K]= & \frac{1}{4} m^{2} \int \mathrm{d}^{4} x \text { e } B^{a b} \square_{2} B_{a b}+\frac{1}{2} \int \mathrm{d}^{4} x e \Phi \square_{0}\left(\square_{0}-m^{2}\right) \Phi \\
& -\frac{1}{2} \int \mathrm{d}^{4} x e \Psi \square_{0}\left(\square_{0}-m^{2}\right) \Psi
\end{aligned}
$$

Then, taking into account (3.16) leads to (3.14). 


\section{Massive super $p$-forms in four dimensions}

In this section we study effective actions of the following massive locally supersymmetric theories in four dimensions: (i) vector multiplet; (ii) tensor multiplet; and (iii) three-form multiplet. In the massless case, these multiplets are naturally described in terms of super $p$-forms, with $p=1,2$ and 3 , respectively. The models for massive vector and tensor multiplets are classically equivalent. Here we will demonstrate their quantum equivalence.

At the component level, the locally supersymmetric models of our interest contain the massive $p$-form models we have studied in the previous section.

\subsection{Setup}

The massive vector multiplet in a supergravity background $[34,66]$ is described in terms of a real scalar prepotential $V$. The action is

$$
S_{\text {vector }}^{(m)}[V]=\frac{1}{2} \int \mathrm{d}^{4} x \mathrm{~d}^{2} \theta \mathrm{d}^{2} \bar{\theta} E V\left\{\frac{1}{8} \mathcal{D}^{\alpha}\left(\overline{\mathcal{D}}^{2}-4 R\right) \mathcal{D}_{\alpha}+m^{2}\right\} V, \quad \bar{V}=V .
$$

The massive tensor multiplet [34] is described in terms of a covariantly chiral spinor superfield $\Psi_{\alpha}, \overline{\mathcal{D}}_{\dot{\beta}} \Psi_{\alpha}=0$, and its conjugate $\bar{\Psi}_{\dot{\alpha}}$. The action is

$$
S_{\text {tensor }}^{(m)}[\Psi, \bar{\Psi}]=-\frac{1}{2} \int \mathrm{d}^{4} x \mathrm{~d}^{2} \theta \mathrm{d}^{2} \bar{\theta} E(G(\Psi))^{2}-\left\{\frac{m^{2}}{2} \int \mathrm{d}^{4} x \mathrm{~d}^{2} \theta \mathcal{E} \Psi^{2}+\text { c.c. }\right\},
$$

where we have introduced the real superfield

$$
G(\Psi):=\frac{1}{2}\left(\mathcal{D}^{\alpha} \Psi_{\alpha}+\overline{\mathcal{D}}_{\dot{\alpha}} \bar{\Psi}^{\dot{\alpha}}\right),
$$

which is covariantly linear, $\left(\overline{\mathcal{D}}^{2}-4 R\right) G=0$. Similar to the vector multiplet, the massive three-form multiplet is formulated in terms of a real scalar prepotential $V$. The corresponding action is obtained from (1.4) by adding a mass term,

$$
S_{3 \text {-form }}^{(m)}[V]=\frac{1}{2} \int \mathrm{d}^{4} x \mathrm{~d}^{2} \theta \mathrm{d}^{2} \bar{\theta} E V\left\{\left(\mathcal{P}_{+}+\mathcal{P}_{-}\right)^{2}-m^{2}\right\} V,
$$

where the operators $\mathcal{P}_{+}$and $\mathcal{P}_{-}$are defined in (1.3). We recall that $\mathcal{P}_{+} U$ and $\mathcal{P}_{-} U$ are covariantly chiral and antichiral, respectively, for any scalar superfield $U$.

Associated with the above massive models are their effective actions defined by

$$
\begin{aligned}
\mathrm{e}^{\mathrm{i} \Gamma_{\text {vector }}^{(m)}} & =\int[\mathcal{D} V] \mathrm{e}^{\mathrm{i} S_{\text {vector }}^{(m)}[V]}, \\
\mathrm{e}^{\mathrm{i} \Gamma_{\text {tensor }}^{(m)}} & =\int[\mathcal{D} \Psi][\mathcal{D} \bar{\Psi}] \mathrm{e}^{\mathrm{i} S_{\text {tensor }}^{(m)}[\Psi, \bar{\Psi}]}, \\
\mathrm{e}^{\mathrm{i} \Gamma_{3 \text {-form }}^{(m)}} & =\int[\mathcal{D} V] \mathrm{e}^{\mathrm{i} S_{3 \text {-form }}^{(m)}[V]} .
\end{aligned}
$$

There exist alternative representations for the effective actions introduced. They may be derived by making use of Stueckelberg reformulations of the models under consideration. 


\subsection{Quantisation of the massive vector multiplet model}

The Stueckelberg reformulation of the massive vector multiplet model is obtained by replacing

$$
V \rightarrow V+\frac{1}{m}\left(\mathcal{P}_{+}+\mathcal{P}_{-}\right) K, \quad \bar{K}=K
$$

in the action (4.1). The resulting action

$$
\begin{aligned}
S_{\text {vector }}^{(m)}[V, K]=\frac{1}{2} \int \mathrm{d}^{4} x \mathrm{~d}^{2} \theta \mathrm{d}^{2} \bar{\theta} E\left\{\frac{1}{8} V \mathcal{D}^{\alpha}\left(\overline{\mathcal{D}}^{2}-4 R\right) \mathcal{D}_{\alpha} V+m^{2} V^{2}\right. \\
\left.+2 m V\left(\mathcal{P}_{+}+\mathcal{P}_{-}\right) K+K\left(\mathcal{P}_{+}+\mathcal{P}_{-}\right)^{2} K\right\} \\
+
\end{aligned}
$$

is invariant under gauge transformations

$$
\delta_{U} V=\left(\mathcal{P}_{+}+\mathcal{P}_{-}\right) U, \quad \delta_{U} K=-m U, \quad \bar{U}=U .
$$

To quantise the gauge theory with action (4.7), we introduce the gauge fixing

$$
\chi=\left(\mathcal{P}_{+}+\mathcal{P}_{-}\right) V+m K-\mathfrak{F},
$$

with $\mathfrak{F}$ a background real superfield. The gauge variation of $\chi$ is

$$
\delta_{U} \chi=\left(\mathcal{P}_{+}+\mathcal{P}_{-}\right)^{2} U-m^{2} U \equiv \mathfrak{O} U,
$$

and therefore the Faddeev-Popov determinant is

$$
\Delta_{\mathrm{FP}}=\operatorname{det} \mathfrak{O}=\exp \left(-2 \mathrm{i} \Gamma_{3 \text {-form }}^{(m)}\right) .
$$

For the effective action we obtain

$$
\mathrm{e}^{\mathrm{i} \Gamma_{\text {vector }}^{(m)}}=\int[\mathcal{D} V][\mathcal{D} K] \operatorname{det} \mathfrak{O} \delta\left[\left(\mathcal{P}_{+}+\mathcal{P}_{-}\right) V+m K-\mathfrak{F}\right] \mathrm{e}^{\mathrm{i} S_{\text {vector }}^{(m)}[V, K]} .
$$

Averaging the right-hand side over $\mathfrak{F}$ with weight

$$
\exp \left\{-\frac{\mathrm{i}}{2} \int \mathrm{d}^{4} x \mathrm{~d}^{2} \theta \mathrm{d}^{2} \bar{\theta} E \mathfrak{F}^{2}\right\}
$$

we obtain

$$
\exp \left\{\mathrm{i} \Gamma_{\text {vector }}^{(m)}\right\}=\exp \left\{-\mathrm{i} \Gamma_{3 \text {-form }}^{(m)}\right\}\left[\operatorname{det}\left(\square_{\mathrm{v}}^{(R)}-m^{2}\right)\right]^{-\frac{1}{2}},
$$

where we have introduced the operator ${ }^{7}[31,82]$

$$
\begin{aligned}
\square_{\mathrm{v}}^{(R)}= & -\frac{1}{8} \mathcal{D}^{\alpha}\left(\overline{\mathcal{D}}^{2}-4 R\right) \mathcal{D}_{\alpha}+\left(\mathcal{P}_{+}+\mathcal{P}_{-}\right)^{2} \\
= & \mathcal{D}^{a} \mathcal{D}_{a}-\frac{1}{4} G^{\alpha \dot{\alpha}}\left[\mathcal{D}_{\alpha}, \overline{\mathcal{D}}_{\dot{\alpha}}\right]-\frac{1}{4} R\left(\overline{\mathcal{D}}^{2}-4 R\right)-\frac{1}{4} \bar{R}\left(\mathcal{D}^{2}-4 \bar{R}\right) \\
& -\frac{1}{4}\left(\mathcal{D}^{\alpha} R\right) \mathcal{D}_{\alpha}-\frac{1}{4}\left(\overline{\mathcal{D}}_{\dot{\alpha}} \bar{R}\right) \overline{\mathcal{D}}^{\dot{\alpha}}-\frac{1}{4}\left(\mathcal{D}^{2} R\right)-\frac{1}{4}\left(\overline{\mathcal{D}}^{2} \bar{R}\right)+2 R \bar{R} .
\end{aligned}
$$

Our final result (4.14) relates the effective actions (4.5a) and (4.5c).

${ }^{7}$ The d'Alembertian $\square_{\mathrm{v}}^{(R)}$ is a member of the family of operators $\square_{\mathrm{v}}^{(\Phi)}$ introduced in [31, 82], where $\square_{\mathrm{v}}^{(\Phi)}=-\frac{1}{8} \mathcal{D}^{\alpha}\left(\overline{\mathcal{D}}^{2}-4 R\right) \mathcal{D}_{\alpha}+\left\{\mathcal{P}_{+}, \mathcal{P}_{-}\right\}+\Phi \mathcal{P}_{+}+\bar{\Phi} \mathcal{P}_{-}$, with $\Phi$ a chiral scalar. 


\subsection{Quantisation of the massive tensor multiplet model}

The Stueckelberg reformulation of the massive tensor multiplet model, eq. (4.2), is obtained by replacing

$$
\Psi_{\alpha} \rightarrow \Psi_{\alpha}+\frac{\mathrm{i}}{2 m} W_{\alpha}(V), \quad W_{\alpha}=-\frac{1}{4}\left(\overline{\mathcal{D}}^{2}-4 R\right) \mathcal{D}_{\alpha} V, \quad \bar{V}=V
$$

in the action (4.2). This leads to the action

$$
\begin{aligned}
S_{\text {tensor }}^{(m)}[\Psi, \bar{\Psi}, V]= & -\frac{1}{2} \int \mathrm{d}^{4} x \mathrm{~d}^{2} \theta \mathrm{d}^{2} \bar{\theta} E(G(\Psi))^{2}-\left\{\frac{m^{2}}{2} \int \mathrm{d}^{4} x \mathrm{~d}^{2} \theta \mathcal{E} \Psi^{2}+\text { c.c. }\right\} \\
& +\int \mathrm{d}^{4} x \mathrm{~d}^{2} \theta \mathrm{d}^{2} \bar{\theta} E\left\{\frac{1}{16} V \mathcal{D}^{\alpha}\left(\overline{\mathcal{D}}^{2}-4 R\right) \mathcal{D}_{\alpha} V+m V L(\Psi)\right\},
\end{aligned}
$$

where we have introduced the covariantly linear superfield

$$
L(\Psi):=\frac{\mathrm{i}}{2}\left(\mathcal{D}^{\alpha} \Psi_{\alpha}-\overline{\mathcal{D}}_{\dot{\alpha}} \bar{\Psi}^{\dot{\alpha}}\right) .
$$

The action is invariant under gauge transformations

$$
\delta_{K} \Psi_{\alpha}=-\frac{\mathrm{i}}{8}\left(\overline{\mathcal{D}}^{2}-4 R\right) \mathcal{D}_{\alpha} K, \quad \delta_{K} V=-m K, \quad \bar{K}=K .
$$

To quantise the gauge theory with action (4.17), we introduce the gauge fixing

$$
\chi=L(\Psi)-m V-\mathfrak{U},
$$

where $\mathfrak{U}$ is a background real superfield. The gauge variation of $\chi$ is

$$
\delta_{K} \chi=\frac{1}{8} \mathcal{D}^{\alpha}\left(\overline{\mathcal{D}}^{2}-4 R\right) \mathcal{D}_{\alpha} K+m^{2} K \equiv \mathfrak{O} K .
$$

Here $\mathfrak{O}$ is exactly the operator which determines the vector multiplet action (4.1). This means that the Faddeev-Popov determinant is

$$
\Delta_{\mathrm{FP}}=\operatorname{det} \mathfrak{O}=\exp \left(-2 \mathrm{i} \Gamma_{\text {vector }}^{(m)}\right) .
$$

As a result, the effective action can be written in the form

$$
\mathrm{e}^{\mathrm{i} \Gamma_{\text {tensor }}^{(m)}}=\int[\mathcal{D} \Psi][\mathcal{D} \bar{\Psi}][\mathcal{D} V] \operatorname{det} \mathfrak{O} \delta[L(\Psi)-m V-\mathfrak{U}] \mathrm{e}^{\mathrm{i} S_{\text {tensor }}^{(m)}[\Psi, \bar{\Psi}, V]} .
$$

Since the right-hand side of (4.23) is independent of $\mathfrak{U}$, we can average it over $\mathfrak{U}$ with weight

$$
\exp \left\{\frac{\mathrm{i}}{2} \int \mathrm{d}^{4} x \mathrm{~d}^{2} \theta \mathrm{d}^{2} \bar{\theta} E \mathfrak{U}^{2}\right\}
$$

This leads to

$$
\exp \left\{\mathrm{i} \Gamma_{\text {tensor }}^{(m)}\right\}=\exp \left\{-\mathrm{i} \Gamma_{\text {vector }}^{(m)}\right\}\left[\operatorname{det}\left(\square_{\mathrm{c}}-m^{2}\right) \operatorname{det}\left(\square_{\mathrm{a}}-m^{2}\right)\right]^{\frac{1}{2}},
$$

where the d'Alembertian $\square_{\mathrm{c}}$ acts on the space of covariantly chiral spinors $[19,29]$

$$
\begin{aligned}
\square_{\mathrm{c}} \Psi_{\alpha}:= & \frac{1}{16}\left(\overline{\mathcal{D}}^{2}-4 R\right)\left(\mathcal{D}^{2}-6 \bar{R}\right) \Psi_{\alpha} \\
= & \left\{\mathcal{D}^{b} \mathcal{D}_{b}+\frac{1}{4} R \mathcal{D}^{2}+\mathrm{i} G^{b} \mathcal{D}_{b}+\frac{1}{4}\left(\mathcal{D}^{\beta} R\right) \mathcal{D}_{\beta}-\frac{3}{8}\left(\overline{\mathcal{D}}^{2}-4 R\right) \bar{R}\right\} \Psi_{\alpha} \\
& -\left\{W^{\beta}{ }_{\alpha \gamma} \mathcal{D}_{\beta}+\frac{1}{2}\left(\mathcal{D}^{\beta} W_{\alpha \beta \gamma}\right)\right\} \Psi^{\gamma} .
\end{aligned}
$$

Our final result (4.25) relates the effective actions (4.5a) and (4.5b). 


\subsection{Quantisation of the massive three-form multiplet model}

The Stueckelberg reformulation of the massive tensor multiplet model, eq. (4.4), is obtained by replacing

$$
V \rightarrow V+\frac{1}{m} G(\Psi), \quad G(\Psi):=\frac{1}{2}\left(\mathcal{D}^{\alpha} \Psi_{\alpha}+\overline{\mathcal{D}}_{\dot{\alpha}} \bar{\Psi}^{\dot{\alpha}}\right), \quad \overline{\mathcal{D}}_{\dot{\beta}} \Psi_{\alpha}=0 .
$$

The resulting action

$$
\begin{aligned}
S_{3 \text {-form }}^{(m)}[V, \Psi, \bar{\Psi}]= & \frac{1}{2} \int \mathrm{d}^{4} x \mathrm{~d}^{2} \theta \mathrm{d}^{2} \bar{\theta} E V\left\{\left(\mathcal{P}_{+}+\mathcal{P}_{-}\right)^{2}-m^{2}\right\} V \\
& -\int \mathrm{d}^{4} x \mathrm{~d}^{2} \theta \mathrm{d}^{2} \bar{\theta} E\left\{m V G(\Psi)+\frac{1}{2}(G(\Psi))^{2}\right\}
\end{aligned}
$$

is invariant under gauge transformations

$$
\delta_{\lambda} V=\frac{1}{2}\left(\mathcal{D}^{\alpha} \lambda_{\alpha}+\overline{\mathcal{D}}_{\dot{\alpha}} \bar{\lambda}^{\dot{\alpha}}\right), \quad \delta_{\lambda} \Psi_{\alpha}=-m \lambda_{\alpha}, \quad \overline{\mathcal{D}}_{\dot{\beta}} \lambda_{\alpha}=0 .
$$

To quantise the gauge theory with action (4.28), we introduce the gauge condition

$$
\chi_{\alpha}=\frac{1}{2} W_{\alpha}(V)+m \Psi_{\alpha}-\xi_{\alpha}, \quad W_{\alpha}(V):=-\frac{1}{4}\left(\overline{\mathcal{D}}^{2}-4 R\right) \mathcal{D}_{\alpha} V,
$$

where $\xi_{\alpha}$ is a background chiral spinor. The gauge variation of $\chi_{\alpha}$ is

$$
\delta_{\lambda} \chi_{\alpha}=-\frac{1}{8}\left(\overline{\mathcal{D}}^{2}-4 R\right) \mathcal{D}_{\alpha} G(\lambda)-m^{2} \lambda_{\alpha} \equiv \mathfrak{O} \lambda_{\alpha} .
$$

Here $\mathfrak{O}$ is the operator which determines the massive tensor multiplet model (4.2). This means that the Faddeev-Popov super-determinant is

$$
\Delta_{\mathrm{FP}}=[\operatorname{det} \mathfrak{O}]^{-1}=\exp \left(-2 \mathrm{i} \Gamma_{\text {tensor }}^{(m)}\right)
$$

Therefore, the effective action is given by the path integral

$$
\begin{aligned}
\mathrm{e}^{\mathrm{i} \Gamma_{\text {3-form }}^{(m)}=} & \int[\mathcal{D} V][\mathcal{D} \Psi][\mathcal{D} \bar{\Psi}] \operatorname{det} \mathfrak{O} \mathrm{e}^{\mathrm{i} S_{3-\text { form }}^{(m)}[V, \Psi, \bar{\Psi}]} \\
& \times \delta\left[\frac{1}{2} W_{\alpha}(V)+m \Psi_{\alpha}-\xi_{\alpha}\right] \delta\left[\frac{1}{2} \bar{W}_{\dot{\alpha}}(V)+m \bar{\Psi}_{\dot{\alpha}}-\bar{\xi}_{\dot{\alpha}}\right]
\end{aligned}
$$

Since the right-hand side is independent of the chiral spinor $\xi_{\alpha}$ and its conjugate $\bar{\xi}_{\dot{\alpha}}$, we can average over these superfields with weight

$$
\exp \left\{-\frac{\mathrm{i}}{2}\left(\int \mathrm{d}^{4} x \mathrm{~d}^{2} \theta \mathcal{E} \xi^{2}+\text { c.c. }\right)\right\} .
$$

This will lead to the relation

$$
\exp \left\{\mathrm{i} \Gamma_{3 \text {-form }}^{(m)}\right\}=\exp \left\{-\mathrm{i} \Gamma_{\text {tensor }}^{(m)}\right\}\left[\operatorname{det}\left(\square_{\mathrm{v}}^{(R)}-m^{2}\right)\right]^{-\frac{1}{2}},
$$

which connects the effective actions (4.5b) and (4.5c). 


\subsection{Analysis of the results}

We have derived three different relations which connect the three effective actions defined in (4.5). They are given by the equations (4.14), (4.25) and (4.35). These results have nontrivial implications. Firstly, it follows from (4.14) and (4.35) that

$$
\Gamma_{\text {vector }}^{(m)}=\Gamma_{\text {tensor }}^{(m)} .
$$

Therefore, the classically equivalent theories (4.1) and (4.2) remain equivalent at the quantum level. Secondly, making use of (4.25) and (4.36) leads to

$$
\Gamma_{\text {vector }}^{(m)}=\Gamma_{\text {tensor }}^{(m)}=-\frac{\mathrm{i}}{4} \ln \operatorname{det}\left(\square_{\mathrm{c}}-m^{2}\right)-\frac{\mathrm{i}}{4} \ln \operatorname{det}\left(\square_{\mathrm{a}}-m^{2}\right) .
$$

Thirdly, from (4.35) and (4.37) we deduce

$$
\Gamma_{3 \text {-form }}^{(m)}=\frac{\mathrm{i}}{2} \ln \operatorname{det}\left(\square_{\mathrm{v}}^{(R)}-m^{2}\right)+\frac{\mathrm{i}}{4} \ln \operatorname{det}\left(\square_{\mathrm{c}}-m^{2}\right)+\frac{\mathrm{i}}{4} \ln \operatorname{det}\left(\square_{\mathrm{a}}-m^{2}\right) .
$$

The superfield heat kernels corresponding to the operators appearing in (4.37) and (4.38) were studied in [19, 31, 82, 84, 85].

As follows from (4.37), the effective actions $\Gamma_{\text {vector }}^{(m)}$ and $\Gamma_{\text {tensor }}^{(m)}$ coincide, without any topological mismatch. This is due to the use of the Stueckelberg formulation defined by eqs. (4.6) and (4.7). A topological mismatch will emerge if we consider a slightly different Stueckelberg reformulation, which is obtained by replacing the dynamical superfield in (4.1) by the rule

$$
V \rightarrow V+\frac{1}{m}(\Phi+\bar{\Phi}), \quad \overline{\mathcal{D}}_{\dot{\alpha}} \Phi=0
$$

This leads to the action

$$
S_{\text {vector }}^{(m)}[V, \Phi, \bar{\Phi}]=\frac{1}{2} \int \mathrm{d}^{4} x \mathrm{~d}^{2} \theta \mathrm{d}^{2} \bar{\theta} E\left\{\frac{1}{8} V \mathcal{D}^{\alpha}\left(\overline{\mathcal{D}}^{2}-4 R\right) \mathcal{D}_{\alpha} V+(m V+\Phi+\bar{\Phi})^{2}\right\},(
$$

which possesses the gauge freedom

$$
\delta_{\lambda} V=\lambda+\bar{\lambda}, \quad \delta_{\lambda} \Phi=-m \lambda, \quad \overline{\mathcal{D}}_{\dot{\alpha}} \lambda=0
$$

Modulo a purely topological contribution, the functional (4.38) proves to be twice the effective action of a scalar multiplet. To justify this claim, let us consider the following dynamical system

$$
S^{(m)}[V, \Phi, \bar{\Phi}]=\frac{1}{2} \int \mathrm{d}^{4} x \mathrm{~d}^{2} \theta \mathrm{d}^{2} \bar{\theta} E\left\{V\left(\mathcal{P}_{+}+\mathcal{P}_{-}\right)^{2} V+(\Phi+\bar{\Phi})^{2}+2 m V(\Phi+\bar{\Phi})\right\},
$$

where $\Phi$ is a chiral scalar. This model proves to be dual to the massive three-form theory (4.4). The action (4.42) is invariant under gauge transformations

$$
\delta_{\lambda} V=\frac{1}{2}\left(\mathcal{D}^{\alpha} \lambda_{\alpha}+\overline{\mathcal{D}}_{\dot{\alpha}} \bar{\lambda}^{\dot{\alpha}}\right), \quad \overline{\mathcal{D}}_{\dot{\beta}} \lambda_{\alpha}=0
$$


corresponding to the massless three-form multiplet. Quantisation of the reducible gauge theory can be carried out using the method described in [19]. Next, we represent the chiral scalar $\Phi$ in (4.42) as

$$
\Phi=\mathcal{P}_{+} U, \quad \bar{U}=U
$$

Finally, we introduce new variables $K_{ \pm}=\frac{1}{\sqrt{2}}(V \pm U)$. Then the action turns into

$$
\begin{aligned}
S^{(m)}\left[K_{ \pm}\right]=\frac{1}{2} \int \mathrm{d}^{4} x \mathrm{~d}^{2} \theta \mathrm{d}^{2} \bar{\theta} E\{ & \left\{K_{+}\left(\mathcal{P}_{+}+\mathcal{P}_{-}\right)^{2} K_{+}+K_{-}\left(\mathcal{P}_{+}+\mathcal{P}_{-}\right)^{2} K_{-}\right. \\
& \left.+m K_{+}\left(\mathcal{P}_{+}+\mathcal{P}_{-}\right) K_{+}-m K_{-}\left(\mathcal{P}_{+}+\mathcal{P}_{-}\right) K_{-}\right\}
\end{aligned}
$$

This is the three-form counterpart of the theory

$$
\begin{aligned}
S_{\text {scalar }}^{(m)}\left[\Phi_{ \pm}, \bar{\Phi}_{ \pm}\right]= & \frac{1}{2} \int \mathrm{d}^{4} x \mathrm{~d}^{2} \theta \mathrm{d}^{2} \bar{\theta} E\left\{\left(\Phi_{+}+\bar{\Phi}_{+}\right)^{2}+\left(\Phi_{-}+\bar{\Phi}_{-}\right)^{2}\right\} \\
& +\frac{m}{2}\left\{\int \mathrm{d}^{4} x \mathrm{~d}^{2} \theta \mathcal{E}\left(\Phi_{+}^{2}-\Phi_{-}^{2}\right)+\text { c.c. }\right\},
\end{aligned}
$$

which describes two decoupled massive scalar multiplets in a supergravity background. The quantum effective action for this theory is

$$
\Gamma_{\text {scalar }}^{(m)}=\frac{\mathrm{i}}{2} \ln \left(\operatorname{Det} H^{(R+m)} \operatorname{Det} H^{(R-m)}\right),
$$

where $H^{(\psi)}$ denotes the following operator [31, 82]

$$
H^{(\psi)}=\left(\begin{array}{cc}
\psi & \mathcal{P}_{+} \\
\mathcal{P}_{-} & \bar{\psi}
\end{array}\right), \quad \overline{\mathcal{D}}_{\dot{\alpha}} \psi=0 .
$$

By definition, the operator $H^{(\psi)}$ acts on the space of chiral-antichiral column-vectors

$$
H^{(\psi)}\left(\begin{array}{c}
\eta \\
\bar{\eta}
\end{array}\right)=\left(\begin{array}{c}
\psi \eta+\mathcal{P}_{+} \bar{\eta} \\
\bar{\psi} \bar{\eta}+\mathcal{P}_{-} \eta
\end{array}\right), \quad \overline{\mathcal{D}}^{\dot{\alpha}} \eta=0 .
$$

A useful expression for Det $H^{(\psi)}$ in terms of the functional determinants of covariant d'Alembertians is derived in [31, 82].

Since the effective actions (4.38) and (4.47) should differ only by a topological term, we conclude that

$$
\begin{aligned}
-2 X^{(m)}= & \mathrm{i} \ln \operatorname{det}\left(\square_{\mathrm{v}}^{(R)}-m^{2}\right)+\frac{\mathrm{i}}{2} \ln \operatorname{det}\left(\square_{\mathrm{c}}-m^{2}\right)+\frac{\mathrm{i}}{2} \ln \operatorname{det}\left(\square_{\mathrm{a}}-m^{2}\right) \\
& -\mathrm{i} \ln \left(\operatorname{Det} H^{(R+m)} \operatorname{Det} H^{(R-m)}\right)
\end{aligned}
$$

is a topological invariant. It is a generalisation of the invariant introduced in [19, 29].

Our analysis in this section provides the supersymmetric completion of the results obtained in section 3 . 


\section{$5 \quad$ Discussion and generalisations}

In this paper we derived compact expressions for the massive $p$-form effective actions for $0 \leq p \leq d-1$, where $d$ is the dimension of curved spacetime. We then demonstrated that the effective actions $\Gamma_{p}^{(m)}$ and $\Gamma_{d-p-1}^{(m)}$ differ by a topological invariant. These results were extended to the case of massive super $p$-forms coupled to background $\mathcal{N}=1$ supergravity in four dimensions. There are several interesting $p$-form models which we have not considered in this work and which deserve further studies. Here we briefly discuss such models.

As a natural generalisation of the Cremmer-Scherk model for massive spin- 1 in $d=$ 4 [3], the dynamics of a massive $p$-form in $d$ dimensions can be described in terms of a gauge-invariant action involving two fields $B_{p}$ and $A_{q}$, with $q=d-p-1$, and a topological $(B \wedge F)$ mass term. The action is

$$
\begin{aligned}
S^{(m)}\left[B_{p}, A_{q}\right]=-\frac{1}{2} \int \mathrm{d}^{d} x e\left\{\frac{1}{(p+1) !}\right. & F^{a(p+1)}(B) F_{a(p+1)}(B) \\
& \left.+\frac{1}{(q+1) !} F^{a(q+1)}(A) F_{a(q+1)}(A)\right\}+I^{(m)}\left[B_{p}, A_{q}\right],
\end{aligned}
$$

where $I^{(m)}$ stands for the topological mass term

$$
\begin{aligned}
I^{(m)}\left[B_{p}, A_{q}\right] & =\frac{m}{q !(p+1) !} \int \mathrm{d}^{d} x e \varepsilon^{a(q) b(p+1)} A_{a(q)} F_{b(p+1)}(B) \\
& =(-1)^{d(d-p)} \frac{m}{p !(d-p) !} \int \mathrm{d}^{d} x e \varepsilon^{a(p) b(q+1)} B_{a(p)} F_{b(q+1)}(A) .
\end{aligned}
$$

As is well known, this model is dual to the massive theories $S_{p}^{(m)}[B]$ and $S_{q}^{(m)}[A]$, with $S_{p}^{(m)}[B]$ defined by eq. (2.1). The corresponding duality transformations are described, for completeness, in appendix D.

The action (5.1) is invariant under gauge transformations

$$
\delta_{\zeta} B_{a(p)}=p \nabla_{\left[a_{1}\right.} \zeta_{\left.a_{2} \ldots a_{p}\right]}, \quad \delta_{\xi} A_{a(q)}=q \nabla_{\left[a_{1}\right.} \xi_{\left.a_{2} \ldots a_{q}\right]} .
$$

The corresponding generators are linearly dependent, and therefore the gauge theory (5.1) should be quantised using the Batalin-Vilkovisky formalism [14] or the simpler quantisation schemes [17-19], which are specifically designed to quantise Abelian gauge theories. It would be interesting to show that the effective action for the gauge theory (5.1) coincides with (2.23) modulo a topological invariant.

In four dimensions, a supersymmetric generalisation of the Cremmer-Scherk model was given by Siegel [34]

$$
S^{(m)}[\Psi, \bar{\Psi}, V]=-\frac{1}{2} \int \mathrm{d}^{4} x \mathrm{~d}^{2} \theta \mathrm{d}^{2} \bar{\theta} E\left\{(G(\Psi))^{2}-\frac{1}{8} V \mathcal{D}^{\alpha}\left(\overline{\mathcal{D}}^{2}-4 R\right) \mathcal{D}_{\alpha} V\right\}+I^{(m)},
$$

where the mass term is given by

$$
I^{(m)}[\Psi, \bar{\Psi}, V]=m \int \mathrm{d}^{4} x \mathrm{~d}^{2} \theta \mathrm{d}^{2} \bar{\theta} E V G(\Psi)=-\frac{1}{2} m \int \mathrm{d}^{4} x \mathrm{~d}^{2} \theta \mathcal{E} \Psi^{\alpha} W_{\alpha}(V)+\text { c.c. }
$$


This is a dual formulation for the models (4.1) and (4.2). The action (5.4) is invariant under combined gauge transformations corresponding to the massless vector and tensor multiplets. This reducible massive gauge theory can be quantised using the method described in [19].

The mass term (5.5) is locally superconformal [53]. For the supergravity formulation used in the present paper, this means that (5.5) is super-Weyl invariant. We recall that a super-Weyl transformation of the covariant derivatives [89, 90] is

$$
\delta_{\Sigma} \mathcal{D}_{\alpha}=\left(\bar{\Sigma}-\frac{1}{2} \Sigma\right) \mathcal{D}_{\alpha}+\mathcal{D}^{\beta} \Sigma M_{\alpha \beta}, \quad \delta_{\Sigma} \overline{\mathcal{D}}_{\dot{\alpha}}=\left(\Sigma-\frac{1}{2} \bar{\Sigma}\right) \overline{\mathcal{D}}_{\dot{\alpha}}+\left(\overline{\mathcal{D}}^{\dot{\beta}} \bar{\Sigma}\right) \bar{M}_{\dot{\alpha} \dot{\beta}}
$$

where the parameter $\Sigma$ is chiral, $\overline{\mathcal{D}}_{\dot{\alpha}} \Sigma=0$, and $M_{\alpha \beta}$ and $\bar{M}_{\dot{\alpha} \dot{\beta}}$ are the Lorentz generators defined as in [31]. Such a transformation acts on the prepotentials $V$ and $\Psi_{\alpha}$ as

$$
\delta_{\Sigma} V=0, \quad \delta_{\Sigma} \Psi_{\alpha}=\frac{3}{2} \Sigma \Psi_{\alpha}
$$

see [31] for the technical details. The mass term (5.5) is the supersymmetric version of the $d=4$ Green-Schwarz anomaly cancellation term.

Another supersymmetric analogue of the Cremmer-Scherk model is described by the action (4.42).

If $d$ is even, $d=2 n$, one can introduce massive $n$-form models with two types of mass terms [67-69],

$$
\begin{aligned}
S_{n}^{(m, e)}[B]=-\frac{1}{2 n !} \int \mathrm{d}^{d} x e\left\{\frac{1}{n+1} F^{a(n+1)}(B) F_{a(n+1)}(B)\right. & \\
& \left.+m^{2} B^{a(n)} B_{a(n)}+m e B^{a(n)} * B_{a(n)}\right\},
\end{aligned}
$$

with $m$ and $e$ constant parameters. Here the second mass term vanishes if $n$ is odd (however, it is non-zero in the case of several $n$-forms [69].) The model (5.8) is known to be dual to $S_{n}^{(M)}[B]$ of the type $(2.1)$, where $M=\sqrt{m^{2}+e^{2}}$. The results of section 2 can naturally be extended to the case of the model (5.8).

Supersymmetric extensions of (5.8) have been discussed in several publications including [69-71]. In particular, the massive tensor multiplet model (4.2) possesses the following generalisation:

$$
S_{\text {tensor }}^{(m, e)}[\Psi, \bar{\Psi}]=-\frac{1}{2} \int \mathrm{d}^{4} x \mathrm{~d}^{2} \theta \mathrm{d}^{2} \bar{\theta} E(G(\Psi))^{2}-\frac{1}{2} m\left\{(m+\mathrm{i} e) \int \mathrm{d}^{4} x \mathrm{~d}^{2} \theta \mathcal{E} \Psi^{2}+\text { c.c. }\right\} .
$$

Quantisation of this model can be carried out using the approach developed in section 4 .

In conclusion, we would like to come back to the important work by Duff and van Nieuwenhuizen [26]. Their argument concerning the quantum non-equivalence of the dual two-form and zero-form models in $d=4$ was based on the different trace anomalies. However, these theories are non-conformal and, therefore, the quantum operator $T^{a}{ }_{a}$ "contains the effects of both classical and quantum breaking and is not equal to the trace 
anomaly" [29]. Nevertheless, the argument given in [26] can be refined within a Weylinvariant formulation for general gravity-matter systems $[86,87]$. We recall that a Weyl transformation acts on the covariant derivative as

$$
\nabla_{a} \rightarrow \nabla_{a}^{\prime}=\mathrm{e}^{\sigma}\left(\nabla_{a}+\nabla^{b} \sigma M_{b a}\right)
$$

with the parameter $\sigma(x)$ being arbitrary. Such a transformation is induced by that of the gravitational field

$$
e_{a}^{m} \rightarrow \mathrm{e}^{\sigma} e_{a}{ }^{m} \quad \Longrightarrow \quad g_{m n} \rightarrow \mathrm{e}^{-2 \sigma} g_{m n} .
$$

In the Weyl-invariant formulation for gravity in $d \neq 2$ dimensions, the gravitational field is described in terms of two gauge fields. One of them is the vielbein $e_{m}{ }^{a}(x)$ and the other is a conformal compensator $\varphi(x)$. The latter is a nowhere vanishing scalar field with the Weyl transformation law

$$
\varphi \rightarrow \varphi^{\prime}=\mathrm{e}^{\frac{1}{2}(d-2) \sigma} \varphi .
$$

Any dynamical system is required to be invariant under these transformations. In particular, the Weyl-invariant extension of the Einstein-Hilbert gravity action is

$$
S_{\mathrm{GR}}=\frac{1}{2} \int \mathrm{d}^{d} x e\left\{\nabla^{a} \varphi \nabla_{a} \varphi+\frac{1}{4} \frac{d-2}{d-1} R \varphi^{2}\right\} .
$$

Choosing the Weyl gauge $\varphi=\frac{2}{\kappa} \sqrt{\frac{d-1}{d-2}}$ reduces (5.13) to the Einstein-Hilbert action.

The Weyl-invariant reformulation of the massless $p$-form action (B.1) is

$$
S_{p}[B ; \varphi]=-\frac{1}{2(p+1) !} \int \mathrm{d}^{d} x e \varphi^{2 \Delta_{p}} F^{a(p+1)}(B) F_{a(p+1)}(B), \quad \Delta_{p}=1-\frac{2 p}{d-2} .
$$

Here we have made use of the Weyl transformation ${ }^{8}$ of $B_{p}$

$$
B_{a(p)}^{\prime}=\mathrm{e}^{p \sigma} B_{a(p)} .
$$

Let $\Gamma_{p}[\varphi]$ be the effective action corresponding to (5.14). Unlike the classical action (5.14), the nonlocal functional $\Gamma_{p}[\varphi]$ is not Weyl invariant. However, this Weyl anomaly can be eliminated by adding a local counterterm which depends on the compensator $\varphi$, see [88] for the technical details.

At the classical level, the two massless theories $S_{p}[B ; \varphi]$ and $S_{d-p-2}[A ; \varphi]$ prove to be dual, with $\Delta_{p}=-\Delta_{d-p-2}$. In the even-dimensional case, it was shown in [30] that the quantum effective actions for these theories, $\Gamma_{p}[\varphi]$ and $\Gamma_{d-p-2}[\varphi]$, are related to each other $^{9}$ as

$$
\Gamma_{d-p-2}[\varphi]-\Gamma_{p}[\varphi]-(-1)^{p} \mathfrak{X}^{(m)} \propto \int \mathrm{d}^{d} x e \ln \varphi \mathcal{E}_{d}
$$

\footnotetext{
${ }^{8}$ There are two representations for the $p$-form, $B_{p}=\frac{1}{p !} B_{m_{1} \ldots m_{p}} \mathrm{~d} x^{m_{1}} \wedge \cdots \wedge x^{m_{p}}=\frac{1}{p !} B_{a_{1} \ldots a_{p}} e^{a_{1}} \wedge \cdots \wedge e^{a_{p}}$, with $e^{a}=\mathrm{d} x^{m} e_{m}{ }^{a}$ the vielbein. The $p$-form field with world indices, $B_{m(p)}$, is inert under the Weyl transformations.

${ }^{9}$ The field $\phi=-\Delta_{p} \ln \varphi$ was interpreted in [30] as the dilaton.
} 
where $\mathcal{E}_{d}$ is the Euler invariant,

$$
\mathcal{E}_{d}=\frac{1}{(8 \pi)^{n} n !} \varepsilon^{a_{1} b_{1} \ldots a_{n} b_{n}} \varepsilon^{c_{1} d_{1} \ldots c_{n} d_{n}} R_{a_{1} b_{1} c_{1} d_{1}} \ldots R_{a_{n} b_{n} c_{n} d_{n}}, \quad d=2 n
$$

Relation (5.16) is a generalisation of (B.8). The expression on the right-hand side of (5.16) is a local functional and can be removed by adding a local counterterm. This proves the quantum equivalence of the theories.

In a similar manner supergravity in diverse dimensions can be formulated as conformal supergravity coupled to certain compensating supermultiplet(s) [91]. The super-Weylinvariant extensions of the models (1.1) and (1.2) are given (see, e.g., [53]) by

$$
\begin{aligned}
S_{\text {tensor }}\left[\Psi, \bar{\Psi} ; S_{0}, \bar{S}_{0}\right] & =-\frac{1}{2} \int \mathrm{d}^{4} x \mathrm{~d}^{2} \theta \mathrm{d}^{2} \bar{\theta} E \frac{(G(\Psi))^{2}}{S_{0} \bar{S}_{0}}, \\
S_{\text {chiral }}\left[\Phi, \bar{\Phi} ; S_{0}, \bar{S}_{0}\right] & =\frac{1}{2} \int \mathrm{d}^{4} x \mathrm{~d}^{2} \theta \mathrm{d}^{2} \bar{\theta} E S_{0} \bar{S}_{0}(\Phi+\bar{\Phi})^{2},
\end{aligned}
$$

where $S_{0}$ is the chiral compensator, $\overline{\mathcal{D}}_{\dot{\alpha}} S_{0}=0$, corresponding to the old minimal formulation for $\mathcal{N}=1$ supergravity, see [89, 92-95]. By definition, $S_{0}$ is nowhere vanishing and possesses the super-Weyl transformation $\delta_{\Sigma} S_{0}=\Sigma S_{0}$. The matter chiral scalar in (5.18b) is super-Weyl neutral. The models (5.18a) and (5.18b) are classically equivalent. On general grounds, these models should be equivalent at the quantum level. It would be interesting to carry out explicit calculations to check this. It should be pointed out that the actions (5.18a) and (5.18b) lead to non-minimal operators for which the standard superfield heat kernel techniques [31, 82, 84, 85] for computing effective actions do not work. Quantum supersymmetric theories with non-minimal operators were studied in [96, 97].

Our analysis in this paper was restricted to those systems in which the classical action is quadratic in the dynamical fields and therefore the corresponding effective action admits a closed-form expression in terms of the functional determinants of certain operators. In the case of nonlinear theories, such as the following model $[35,53]$

$$
S=\int \mathrm{d}^{4} x \mathrm{~d}^{2} \theta \mathrm{d}^{2} \bar{\theta} E\left\{S_{0} \bar{S}_{0} \mathfrak{F}\left(\frac{G(\Psi)}{S_{0} \bar{S}_{0}}\right)+\frac{1}{16} V \mathcal{D}^{\alpha}\left(\overline{\mathcal{D}}^{2}-4 R\right) \mathcal{D}_{\alpha} V+m V G(\Psi)\right\}
$$

and its duals, it is not possible to obtain simple expressions for the effective action. Nevertheless, the issue of quantum equivalence can still be addressed using the path integral considerations described by Fradkin and Tseytlin [24]. This approach was used in [20] to prove quantum equivalence of the Freedman-Townsend model [22] and the principal chiral $\sigma$-model.

\section{Acknowledgments}

We thank Fernando Quevedo for bringing important references to our attention. SMK is grateful to Ioseph Buchbinder and Jim Gates for email correspondence, and to Dmitri Sorokin for collaboration at an early stage of this project. His work is supported in part by the Australian Research Council, project No. DP200101944. 


\section{A Hodge-de Rham operator}

Given a non-negative integer $p \leq d$, the so-called Hodge-de Rham operator (also known as the covariant d'Alembertian)

$$
\square_{p}=-\left(\mathrm{d}^{\dagger} \mathrm{d}+\mathrm{dd}^{\dagger}\right)
$$

is defined to act on the space of $p$-forms. We recall that the operators of exterior derivative $\mathrm{d}$ and co-derivative $\mathrm{d}^{\dagger}$ are defined to act on a $p$-form $\omega$ as

$$
\begin{aligned}
& \mathrm{d}: \omega_{a_{1} \ldots a_{p}} \rightarrow(\mathrm{d} \omega)_{a_{1} \ldots a_{p+1}}=(p+1) \nabla_{\left[a_{1}\right.} \omega_{\left.a_{2} \ldots a_{p+1}\right]}, \\
& \mathrm{d}^{\dagger}: \quad \omega_{a_{1} \ldots a_{p}} \rightarrow\left(\mathrm{d}^{\dagger} \omega\right)_{a_{1} \ldots a_{p-1}}=-\nabla^{b} \omega_{b a_{1} \ldots a_{p-1}} .
\end{aligned}
$$

These operators are nilpotent, $\mathrm{d}^{2}=0$ and $\left(\mathrm{d}^{\dagger}\right)^{2}=0$, and are adjoint of each other,

$$
\frac{1}{(p+1) !} \int \mathrm{d}^{d} x e(\mathrm{~d} \omega)_{a_{1} \ldots a_{p+1}} \varphi^{a_{1} \ldots a_{p+1}}=\frac{1}{p !} \int \mathrm{d}^{d} x e \omega_{a_{1} \ldots a_{p}}\left(\mathrm{~d}^{\dagger} \varphi\right)^{a_{1} \ldots a_{p}}
$$

with respect to the inner product

$$
\left\langle\omega_{p}, \psi_{p}\right\rangle=\frac{1}{p !} \int \mathrm{d}^{d} x e \omega_{a_{1} \ldots a_{p}} \psi^{a_{1} \ldots a_{p}} .
$$

In the case of a $d$-dimensional curved space $\mathcal{M}^{d}$, the action of $\square_{p}$ on a $p$-form $\omega_{a_{1} \ldots a_{p}}$ can be written as

$$
\square_{p} \omega_{a_{1} \ldots a_{p}}=\nabla^{b} \nabla_{b} \omega_{a_{1} \ldots a_{p}}+\sum_{k=1}^{p}(-1)^{k}\left[\nabla^{b}, \nabla_{a_{k}}\right] \omega_{b a_{1} \ldots \widehat{a_{k} \ldots a_{p}}} .
$$

The Hodge-de Rham operators have the important properties

$$
\mathrm{d} \square_{p}=\square_{p+1} \mathrm{~d}, \quad \mathrm{~d}^{\dagger} \square_{p}=\square_{p-1} \mathrm{~d}^{\dagger},
$$

which are used in section 2 .

\section{B Massless $p$-forms in $d$ dimensions}

Setting $m=0$ in (2.1) gives the massless $p$-form field theory

$$
S_{p}[B]=-\frac{1}{2(p+1) !} \int \mathrm{d}^{d} x \text { e } F^{a_{1} \ldots a_{p+1}}(B) F_{a_{1} \ldots a_{p+1}}(B) .
$$

The field strength $F_{p+1}(B)$ is invariant under gauge transformations

$$
\delta_{\zeta} B_{a(p)}=p \nabla_{\left[a_{1}\right.} \zeta_{\left.a_{2} \ldots a_{p}\right]},
$$

and so is the action. It is known, by Poincaré duality, that the massless gauge theories with actions $S_{p}[B]$ and $S_{d-p-2}[B]$ are classically equivalent. 
The energy-momentum tensor, $T_{p}^{a b}(B)$, corresponding to (B.1) is obtained from (2.5) by setting $m=0$.

$$
T_{p}^{a b}(B)=\frac{1}{p !}\left\{F^{a c_{1} \ldots c_{p}}(B) F_{c_{1} \ldots c_{p}}^{b}(B)-\frac{1}{2(p+1)} \eta^{a b} F^{c_{1} \ldots c_{p+1}}(B) F_{c_{1} \ldots c_{p+1}}(B)\right\} .
$$

It is conserved on-shell, $\nabla_{b} T_{p}^{a b}=0$. If the dimension of spacetime is even $p+1=d / 2$, the energy-momentum tensor is traceless in the massless case,

$$
d=2(p+1) \quad \Longrightarrow \quad \eta_{a b} T_{d / 2-1}^{a b}=0 .
$$

This is a corollary of the fact that the $p$-form action in $2(p+1)$ spacetime dimensions

$$
S_{p}[B]=-\frac{1}{2(p+1) !} \int \mathrm{d}^{2(p+1)} x e F^{a_{1} \ldots a_{p+1}}(B) F_{a_{1} \ldots a_{p+1}}(B)
$$

is invariant under arbitrary Weyl rescaling of the vielbein.

Let $\Gamma_{p}$ denote the effective action for the massless $p$-form theory (B.1). As shown in [18] (see also [17]),

$$
\Gamma_{p}=\frac{\mathrm{i}}{2} \sum_{k=0}^{p}(-1)^{k}(1+k) \ln \operatorname{det} \square_{p-k}
$$

In the case that $p=d-1$, the action describes no local degrees of freedom, and therefore the corresponding effective action should be a topological invariant. Indeed, making use of (B.6) allows us to rewrite $\Gamma_{d-1}$ in the form

$$
\Gamma_{d-1}=-\frac{d}{2} \mathfrak{X}, \quad \mathfrak{X}=\frac{\mathrm{i}}{2} \sum_{k=0}^{d}(-1)^{k} \ln \operatorname{det} \square_{k} .
$$

The functional $\mathfrak{X}$ is obtained from $\mathfrak{X}^{(m)}$ given by eq. (2.30) by setting $m=0$.

For $p \neq d-1, d$, it is known that the massless $p$-form and $(d-p-2)$-form models are classically equivalent. For the corresponding effective actions, the following relation holds:

$$
\Gamma_{d-p-2}-\Gamma_{p}=(-1)^{p}\left(\frac{d}{2}-p-1\right) \mathfrak{X} .
$$

This result was established in $[19,24,29]$ for $d=4$, and later generalised to the $d>4$ case in $[30,98]$.

\section{Massless three-form in four dimensions}

The analysis described in the previous appendix has some nuances in the $d=p+1$ case. For simplicity, here we discuss the massless three-form in four dimensions.

The gauge three-form model is described by the action

$$
\widetilde{S}_{3}[V]=\frac{1}{2} \int_{\mathcal{M}} \mathrm{d}^{4} x e H^{2}-\int_{\mathcal{M}} \mathrm{d}^{4} x e \nabla_{a}\left(V^{a} H\right) \equiv S_{3}[V]-\int_{\mathcal{M}} \mathrm{d}^{4} x e \nabla_{a}\left(V^{a} H\right),
$$


where $H:=\nabla_{a} V^{a}$ is the field strength being invariant under gauge transformations

$$
\delta_{B} V^{a}=\frac{1}{2} \varepsilon^{a b c d} \nabla_{b} B_{c d} .
$$

The second term in the action is a boundary term; it was introduced in [40, 43]. To obtain a consistent variation problem, one demands [40] that

$$
\left.\delta H\right|_{\partial \mathcal{M}}=0
$$

such that an arbitrary variation of the action is

$$
\delta \widetilde{S}_{3}[V]=-\int_{\mathcal{M}} \mathrm{d}^{4} x e \delta V^{a} \nabla_{a} H-\int_{\mathcal{M}} \mathrm{d}^{4} x e \nabla_{a}\left(V^{a} \delta H\right) .
$$

The equation of motion is

$$
\nabla_{a} H=0 \quad \Longrightarrow \quad H=c=\text { const }
$$

This shows that the model under consideration has no local degrees of freedom.

Different values of $c$ correspond to different vacua in the quantum theory. When computing the path integral, for a given $c$ we make use of the background-quantum splitting

$$
V^{a}=V_{0}^{a}+v^{a}, \quad \nabla_{a} V_{0}^{a}=c,
$$

such that the classical action becomes

$$
\widetilde{S}_{3}[V]=-\frac{1}{2} c^{2} \int_{\mathcal{M}} \mathrm{d}^{4} x e+S_{3}[v] .
$$

Here the first contribution on the right is the cosmological term. Evaluating the path integral, for the effective action one gets

$$
\Gamma_{3}\left[g_{m n}\right]=-\frac{1}{2} c^{2} \int_{\mathcal{M}} \mathrm{d}^{4} x e-2 \mathfrak{X},
$$

where we have defined

$$
\mathfrak{X}:=\frac{i}{2} \ln \frac{\operatorname{det} \square_{2}\left[\operatorname{det} \square_{0}\right]^{2}}{\left[\operatorname{det} \square_{1}\right]^{2}}
$$

The functional $\mathfrak{X}$ is the four-dimensional version of the topological invariant (B.7).

\section{Duality with topological mass term}

To construct a dual formulation for (5.1), we introduce the first-order action

$$
\begin{array}{r}
S\left[L_{q}, A_{q}, C_{q-1}\right]=\frac{1}{q !} \int \mathrm{d}^{d} x e\left\{\frac{1}{2} L^{a(q)} L_{a(q)}-\frac{1}{2(q+1)} F^{a(q+1)}(A) F_{a(q+1)}(A)\right. \\
\left.+L^{a(q)}\left[m A_{a(q)}+F_{a(q)}(C)\right]\right\}
\end{array}
$$


where $L_{a(q)}$ and $C_{a(q-1)}$ are unconstrained antisymmetric tensor fields. The equation of motion for $C_{a(q-1)}$ implies that $L^{a(q)}=\frac{1}{(p+1) !} \varepsilon^{a(q) b(p+1)} F_{b(p+1)}(B)$, and then the action (D.1) turns into (5.1). On the other hand, we can eliminate $L_{a(q)}$ from the action (D.1) using the corresponding equation of motion. This leads to

$$
\begin{array}{r}
S_{q}^{(m)}\left[A_{q}, C_{q-1}\right]=-\frac{1}{2 q !} \int \mathrm{d}^{d} x e\left\{\frac{1}{q+1} F^{a(q+1)}(A) F_{a(q+1)}(A)\right. \\
\left.+\left[m A_{a(q)}+F_{a(q)}(C)\right]^{2}\right\},
\end{array}
$$

This is the Stueckelberg formulation for the massive $(d-p-1)$-form model, see eq. (2.13). Thus we have shown that the massive $q$-form model (D.2) is dual to (5.1).

There is an alternative dual formulation for (5.1), which is obtained by making use of the first-order action

$$
\begin{array}{r}
S\left[L_{p}, B_{p}, V_{p-1}\right]=\frac{1}{p !} \int \mathrm{d}^{d} x e\left\{\frac{1}{2} L^{a(p)} L_{a(p)}-\frac{1}{2(p+1)} F^{a(p+1)}(B) F_{a(p+1)}(B)\right. \\
\left.+(-1)^{d+d p} L^{a(p)}\left[m B_{a(p)}+F_{a(p)}(V)\right]\right\},
\end{array}
$$

where $L_{a(p)}$ and $V_{a(p-1)}$ are unconstrained antisymmetric tensor fields. The equation of motion for $V_{a(p-1)}$ implies that $L^{a(p)}=\frac{1}{(q+1) !} \varepsilon^{a(p) b(q+1)} F_{b(q+1)}(A)$, and then the action (D.3) turns into (5.1). On the other hand, integrating out $L_{a(p)}$ leads to the massive $p$-form model (2.13).

Open Access. This article is distributed under the terms of the Creative Commons Attribution License (CC-BY 4.0), which permits any use, distribution and reproduction in any medium, provided the original author(s) and source are credited.

\section{References}

[1] V.I. Ogievetsky and I.V. Polubarinov, The notoph and its possible interactions, Sov. J. Nucl. Phys. 4 (1967) 156 [Yad. Fiz. 4 (1966) 216] [INSPIRE]; reprinted in Supersymmetries and quantum symmetries, J. Wess and E. A. Ivanov eds., Springer, Germany (1999).

[2] M. Kalb and P. Ramond, Classical direct interstring action, Phys. Rev. D 9 (1974) 2273 [INSPIRE].

[3] E. Cremmer and J. Scherk, Spontaneous dynamical breaking of gauge symmetry in dual models, Nucl. Phys. B 72 (1974) 117.

[4] F. Gliozzi, J. Scherk and D.I. Olive, Supersymmetry, supergravity theories and the dual spinor model, Nucl. Phys. B 122 (1977) 253 [INSPIRE].

[5] E. Cremmer, J. Scherk and S. Ferrara, SU(4) invariant supergravity theory, Phys. Lett. B $\mathbf{7 4}$ (1978) 61 [INSPIRE].

[6] E. Cremmer, B. Julia and J. Scherk, Supergravity theory in eleven-dimensions, Phys. Lett. B 76 (1978) 409 [INSPIRE].

[7] P. Van Nieuwenhuizen, Supergravity, Phys. Rept. 68 (1981) 189 [InSPIRE]. 
[8] A. Salam and E. Sezgin, Supergravities in diverse dimensions, World Scientific, Singapore (1989).

[9] Y. Tanii, Introduction to supergravity, Springer, Germany (2014).

[10] M.B. Green, J.H. Schwarz and E. Witten, Superstring theory, Cambridge University Press, Cambridge U.K. (1987).

[11] J. Polchinski, String theory, Cambridge University Press, Cambridge U.K. (1998).

[12] K. Becker, M. Becker and J.H. Schwarz, String theory and M-theory: a modern introduction, Cambridge University Press, Cambridge U.K. (2007).

[13] R. Blumenhagen, D. Lüst and S. Theisen, Basic concepts of string theory, Springer, Germany (2013).

[14] I.A. Batalin and G.A. Vilkovisky, Quantization of gauge theories with linearly dependent generators, Phys. Rev. D 28 (1983) 2567 [Erratum ibid. 30 (1984) 508] [INSPIRE].

[15] A.S. Schwarz, The partition function of degenerate quadratic functional and Ray-Singer invariants, Lett. Math. Phys. 2 (1978) 247 [INSPIRE].

[16] A.S. Schwarz, The partition function of a degenerate functional, Commun. Math. Phys. 67 (1979) 1 [INSPIRE].

[17] W. Siegel, Hidden ghosts, Phys. Lett. B 93 (1980) 170 [INSPIRE].

[18] Y. Obukhov, The geometrical approach to antisymmetric tensor field theory, Phys. Lett. B 109 (1982) 195 [INSPIRE].

[19] I.L. Buchbinder and S.M. Kuzenko, Quantization of the classically equivalent theories in the superspace of simple supergravity and quantum equivalence, Nucl. Phys. B 308 (1988) 162 [INSPIRE].

[20] I.L. Buchbinder and S.M. Kuzenko, Quantum equivalence of the Freedman-Townsend model and the principal chiral $\sigma$-model, unpublished (1987).

[21] S.P. de Alwis, M.T. Grisaru and L. Mezincescu, Quantization and unitarity in antisymmetric tensor gauge theories, Nucl. Phys. B 303 (1988) 57 [INSPIRE].

[22] D.Z. Freedman and P.K. Townsend, Antisymmetric tensor gauge theories and nonlinear $\sigma$-models, Nucl. Phys. B 177 (1981) 282 [INSPIRE].

[23] S.E. Hjelmeland and U. Lindström, Duality for the nonspecialist, hep-th/9705122 [INSPIRE].

[24] E.S. Fradkin and A.A. Tseytlin, Quantum equivalence of dual field theories, Annals Phys. 162 (1985) 31 [INSPIRE].

[25] E. Sezgin and P. van Nieuwenhuizen, Renormalizability properties of antisymmetric tensor fields coupled to gravity, Phys. Rev. D 22 (1980) 301 [INSPIRE].

[26] M.J. Duff and P. van Nieuwenhuizen, Quantum inequivalence of different field representations, Phys. Lett. B 94 (1980) 179 [INSPIRE].

[27] M.J. Duff, Antisymmetric tensors and supergravity, in Superspace and Supergravity, S.W. Hawking and M. Roček eds., Cambridge University Press, Cambridge U.K. (1981).

[28] W. Siegel, Quantum equivalence of different field representations, Phys. Lett. B 103 (1981) 107 [INSPIRE]. 
[29] M.T. Grisaru, N.K. Nielsen, W. Siegel and D. Zanon, Energy momentum tensors, supercurrents, (super)traces and quantum Equivalence, Nucl. Phys. B 247 (1984) 157 [INSPIRE].

[30] P.B. Gilkey, K. Kirsten, D. Vassilevich and A. Zelnikov, Duality symmetry of the p form effective action and supertrace of the twisted de Rham complex, Nucl. Phys. B 648 (2003) 542 [hep-th/0209125] [INSPIRE].

[31] I.L. Buchbinder and S.M. Kuzenko, Ideas and methods of supersymmetry and supergravity or a walk through superspace, IOP, Bristol, U.K. (1995).

[32] S.J. Gates Jr., Super p form gauge superfields, Nucl. Phys. B 184 (1981) 381 [InSPIRE].

[33] S.J. Gates Jr., M.T. Grisaru, M. Roček and W. Siegel, Superspace or one thousand and one lessons in supersymmetry, Frontiers in Physics Volume 58, Springer, Germany (1983) [hep-th/0108200] [INSPIRE].

[34] W. Siegel, Gauge spinor superfield as a scalar multiplet, Phys. Lett. B 85 (1979) 333 [INSPIRE].

[35] U. Lindström and M. Roček, Scalar tensor duality and $N=1, N=2$ nonlinear $\sigma$-models, Nucl. Phys. B 222 (1983) 285 [inSPIRE].

[36] B. de Wit and M. Roček, Improved tensor multiplets, Phys. Lett. B 109 (1982) 439 [INSPIRE].

[37] V. Ogievetsky and E. Sokatchev, Equation of motion for the axial gravitational superfield, Sov. J. Nucl. Phys. 32 (1980) 589 [Yad. Fiz. 32 (1980) 1142] [inSPIRE].

[38] A. Aurilia, H. Nicolai and P.K. Townsend, Hidden constants: the theta parameter of QCD and the cosmological constant of $N=8$ supergravity, Nucl. Phys. B 176 (1980) 509 [INSPIRE].

[39] M.J. Duff, The cosmological constant is possibly zero, but the proof is probably wrong, Phys. Lett. B 226 (1989) 36 [Conf. Proc. C 8903131 (1989) 403] [InSPIRE].

[40] M.J. Duncan and L.G. Jensen, Four forms and the vanishing of the cosmological constant, Nucl. Phys. B 336 (1990) 100 [InSPIRE].

[41] R. Bousso and J. Polchinski, Quantization of four form fluxes and dynamical neutralization of the cosmological constant, JHEP 06 (2000) 006 [hep-th/0004134] [INSPIRE].

[42] S.M. Kuzenko, Nilpotent $\mathcal{N}=1$ tensor multiplet, JHEP 04 (2018) 131 [arXiv:1712.09258] [INSPIRE].

[43] K. Groh, J. Louis and J. Sommerfeld, Duality and couplings of 3-form-multiplets in $N=1$ supersymmetry, JHEP 05 (2013) 001 [arXiv: 1212.4639] [INSPIRE].

[44] F. Farakos, S. Lanza, L. Martucci and D. Sorokin, Three-forms in Supergravity and Flux Compactifications, Eur. Phys. J. C 77 (2017) 602 [arXiv:1706.09422] [InSPIRE].

[45] I. Bandos, F. Farakos, S. Lanza, L. Martucci and D. Sorokin, Three-forms, dualities and membranes in four-dimensional supergravity, JHEP 07 (2018) 028 [arXiv:1803.01405] [INSPIRE].

[46] N. Kemmer, On the theory of particles of spin 1, Helv. Phys. Acta 33 (1960) 829.

[47] Y. Takahashi and R. Palmer, Gauge-independent formulation of a massive field with spin one, Phys. Rev. D 1 (1970) 2974 [InSPIRE].

[48] T.L. Curtright and P.G.O. Freund, Massive dual fields, Nucl. Phys. B 172 (1980) 413 [INSPIRE]. 
[49] T. Curtright, Generalized gauge fields, Phys. Lett. B 165 (1985) 304 [INSPIRE].

[50] S. Deser and E. Witten, Dynamical properties of antisymmetric tensor fields, Nucl. Phys. B 178 (1981) 491 [INSPIRE].

[51] P.K. Townsend, Classical properties of antisymmetric tensor gauge fields, in Gauge field theories: theoretical studies and computer simulations, W. Giarczynski ed., Harwood Academic, U.K. (1981).

[52] O. Foda, Nonplanar loops leave the Veneziano model photon massless, Phys. Lett. B 188 (1987) 324 [INSPIRE].

[53] S. Cecotti, S. Ferrara and L. Girardello, Massive vector multiplets from superstrings, Nucl. Phys. B 294 (1987) 537 [INSPIRE].

[54] J. Louis and A. Micu, Type 2 theories compactified on Calabi-Yau threefolds in the presence of background fluxes, Nucl. Phys. B 635 (2002) 395 [hep-th/0202168] [INSPIRE].

[55] T.W. Grimm and J. Louis, The effective action of $N=1$ Calabi-Yau orientifolds, Nucl. Phys. B 699 (2004) 387 [hep-th/0403067] [INSPIRE].

[56] F. Bastianelli, P. Benincasa and S. Giombi, Worldline approach to vector and antisymmetric tensor fields, JHEP 04 (2005) 010 [hep-th/0503155] [INSPIRE].

[57] F. Bastianelli, P. Benincasa and S. Giombi, Worldline approach to vector and antisymmetric tensor fields. II., JHEP 10 (2005) 114 [hep-th/0510010] [INSPIRE].

[58] I.L. Buchbinder, E.N. Kirillova and N.G. Pletnev, Quantum equivalence of massive antisymmetric tensor field models in curved space, Phys. Rev. D 78 (2008) 084024 [arXiv:0806.3505] [INSPIRE].

[59] C.P. Burgess, J.P. Derendinger, F. Quevedo and M. Quirós, On gaugino condensation with field dependent gauge couplings, Annals Phys. 250 (1996) 193 [hep-th/9505171] [INSPIRE].

[60] G.R. Farrar, G. Gabadadze and M. Schwetz, On the effective action of $N=1$ supersymmetric Yang-Mills theory, Phys. Rev. D 58 (1998) 015009 [hep-th/9711166] [InSPIRE].

[61] D.G. Cerdeno, A. Knauf and J. Louis, A note on effective $N=1$ super-Yang-Mills theories versus lattice results, Eur. Phys. J. C 31 (2003) 415 [hep-th/0307198] [INSPIRE].

[62] I. Bandos, S. Lanza and D. Sorokin, Supermembranes and domain walls in $\mathcal{N}=1, D=4$ SYM, JHEP 12 (2019) 021 [Erratum ibid. 05 (2020) 031] [arXiv:1905.02743] [INSPIRE].

[63] E. Dudas, Three-form multiplet and Inflation, JHEP 12 (2014) 014 [arXiv:1407.5688] [INSPIRE].

[64] S. Lanza, F. Marchesano, L. Martucci and D. Sorokin, How many fluxes fit in an EFT?, JHEP 10 (2019) 110 [arXiv:1907.11256] [INSPIRE].

[65] S. Lanza, Exploring the Landscape of effective field theories, arXiv:1912.08935 [INSPIRE].

[66] A. Van Proeyen, Massive vector multiplets in supergravity, Nucl. Phys. B 162 (1980) 376 [INSPIRE].

[67] F. Quevedo and C.A. Trugenberger, Phases of antisymmetric tensor field theories, Nucl. Phys. B 501 (1997) 143 [hep-th/9604196] [inSPIRE].

[68] M.C. Diamantini, F. Quevedo and C.A. Trugenberger, Confining string with topological term, Phys. Lett. B 396 (1997) 115 [hep-th/9612103] [INSPIRE]. 
[69] R. D'Auria and S. Ferrara, Dyonic masses from conformal field strengths in D even dimensions, Phys. Lett. B 606 (2005) 211 [hep-th/0410051] [INSPIRE].

[70] J. Louis and W. Schulgin, Massive tensor multiplets in $N=1$ supersymmetry, Fortsch. Phys. 53 (2005) 235 [hep-th/0410149] [INSPIRE].

[71] S.M. Kuzenko, On massive tensor multiplets, JHEP 01 (2005) 041 [hep-th/0412190] [INSPIRE].

[72] S.J. Gates Jr. and W. Siegel, Variant superfield representations, Nucl. Phys. B 187 (1981) 389 [INSPIRE].

[73] R. Grimm, J. Wess and B. Zumino, A complete solution of the Bianchi identities in superspace, Nucl. Phys. B 152 (1979) 255 [InSPIRE].

[74] J. Wess and B. Zumino, Superfield Lagrangian for supergravity, Phys. Lett. B 74 (1978) 51 [INSPIRE].

[75] J. Wess and J. Bagger, Supersymmetry and supergravity, Princeton University Press, Princeton U.S.A. (1992).

[76] W. Siegel, Supergravity superfields without a supermetric, Harvard preprint HUTP-77/A068 (1977).

[77] W. Siegel, A polynomial action for a massive, self-interacting chiral superfield coupled to supergravity, Harvard preprint HUTP-77/A077 (1977).

[78] W. Siegel, The superfield supergravity action, Harvard preprint HUTP-77/A080 (1977).

[79] W. Siegel, A derivation of the supercurrent superfield, Harvard preprint HUTP-77/A089 (1977).

[80] K.S. Stelle and P.C. West, Minimal auxiliary fields for supergravity, Phys. Lett. B 74 (1978) 330 [INSPIRE].

[81] S. Ferrara and P. van Nieuwenhuizen, The auxiliary fields of supergravity, Phys. Lett. B 74 (1978) 333 [INSPIRE].

[82] I.L. Buchbinder and S.M. Kuzenko, Matter superfields in external supergravity: Green functions, effective action and superconformal anomalies, Nucl. Phys. B 274 (1986) 653 [INSPIRE].

[83] S. Rosenberg, The Laplacian on a Riemannian manifold, London Mathematical Society Student Texts 31, Cambridge University Press, Cambridge U.K. (1998).

[84] I.N. McArthur, Super b(4) coefficients, Phys. Lett. B 128 (1983) 194 [INSPIRE].

[85] I.N. McArthur, Super b(4) coefficients in supergravity, Class. Quant. Grav. 1 (1984) 245.

[86] S. Deser, Scale invariance and gravitational coupling, Annals Phys. 59 (1970) 248 [INSPIRE].

[87] B. Zumino, Effective Lagrangians and broken symmetries, in Lectures on elementary particles and quantum field theory. Volume 2, S. Deser et al. eds., MIT PRess, U.S.A. (1970).

[88] B. de Wit and M.T. Grisaru, Compensating fields and anomalies, in Quantum field theory and quantum statistics. Volume 2, I.A. Batalin et al. eds., Adam Hilger, Bristol U.K. (1987).

[89] W. Siegel, Solution to constraints in Wess-Zumino supergravity formalism, Nucl. Phys. B 142 (1978) 301 [INSPIRE]. 
[90] P.S. Howe and R.W. Tucker, Scale invariance in superspace, Phys. Lett. B 80 (1978) 138 [INSPIRE].

[91] M. Kaku and P.K. Townsend, Poincaré supergravity as broken superconformal gravity, Phys. Lett. B 76 (1978) 54 [inSPIRE].

[92] W. Siegel and S.J. Gates Jr., Superfield supergravity, Nucl. Phys. B 147 (1979) 77 [inSPIRE].

[93] T. Kugo and S. Uehara, Improved superconformal gauge conditions in the $N=1$ supergravity Yang-Mills matter system, Nucl. Phys. B 222 (1983) 125 [inSPIRE].

[94] S. Ferrara, L. Girardello, T. Kugo and A. Van Proeyen, Relation between different auxiliary field formulations of $N=1$ supergravity coupled to matter, Nucl. Phys. B 223 (1983) 191 [INSPIRE].

[95] T. Kugo and S. Uehara, Conformal and Poincaré tensor calculi in $N=1$ supergravity, Nucl. Phys. B 226 (1983) 49 [INSPIRE].

[96] I.L. Buchbinder, S.M. Kuzenko and O.A. Solovev, One loop counterterms of Wess-Zumino model in the $N=1$ nonminimal supergravity background, Nucl. Phys. B 322 (1989) 277 [INSPIRE].

[97] S.M. Kuzenko, Non-compact duality, super-Weyl invariance and effective actions, JHEP 07 (2020) 222 [arXiv : 2006. 00966] [InSPIRE].

[98] J.L.F. Barbón, Generalized Abelian S duality and coset constructions, Nucl. Phys. B 452 (1995) 313 [hep-th/9506137] [INSPIRE]. 